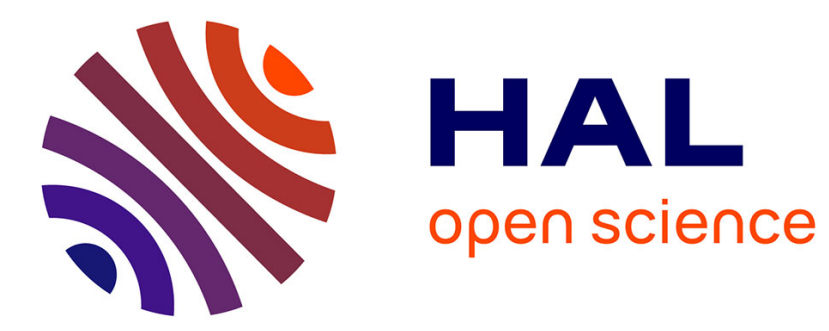

\title{
Aux racines de la rose: Louis du Périer, consul et bibliophile lyonnais
}

\author{
Jean-Benoît Krumenacker
}

\section{To cite this version:}

Jean-Benoît Krumenacker. Aux racines de la rose: Louis du Périer, consul et bibliophile lyonnais. Revue historique, 2019, 3 (691), pp.629-677. hal-02541710

\section{HAL Id: hal-02541710 https://hal-amu.archives-ouvertes.fr/hal-02541710}

Submitted on 14 May 2020

HAL is a multi-disciplinary open access archive for the deposit and dissemination of scientific research documents, whether they are published or not. The documents may come from teaching and research institutions in France or abroad, or from public or private research centers.
L'archive ouverte pluridisciplinaire HAL, est destinée au dépôt et à la diffusion de documents scientifiques de niveau recherche, publiés ou non, émanant des établissements d'enseignement et de recherche français ou étrangers, des laboratoires publics ou privés. 


\section{Aux racines de la rose : Louis du Périer, consul et bibliophile lyonnais}

JEAN-BENOIT KRUMENACKER

« Ta douleur, Du Périer, sera donc éternelle »? Par cette interrogation, en un vers de la toute fin du XVI ${ }^{\mathrm{e}}$ siècle, François de Malherbe faisait entrer dans l'histoire de la littérature française ${ }^{1}$ le nom de cette famille et la rendait populaire chez des générations d'écoliers jusqu'à aujourd'hui. Cette famille du Périer n'est pas complètement obscure et est même plutôt bien connue à l'époque moderne où elle fait partie de la noblesse de robe provençale. Mais les mérites de ses seuls membres ne la font guère briller que dans une histoire très locale parmi les centaines de familles de la noblesse et de la bourgeoisie de l'ancien comté de Provence. Par un de ces curieux hasards de l'histoire, ce ne sont pas les offices auprès du Parlement d'Aix, ni les marquisats, les victoires militaires ou tant de ces honneurs âprement conquis par les différentes générations des du Périer qui ont inscrit leur famille dans l'Histoire mais la mort de la petite Marguerite à l'âge de cinq ans, le désespoir d'un père et la fidélité d'un $a^{2} i^{2}$. Outre ce drame familial qui permet de mesurer l'entourage littéraire de François du Périer, peu de choses ont été dites sur cette famille. Comme la plupart des familles de la noblesse d'Ancien Régime, on la retrouve dans les nobiliaires et généalogies qui présentent ses origines, largement fantasmées ${ }^{3}$ dès que l'on remonte au-delà du personnage que nous mettrons au centre de cet article : Louis du Périer. Ce dernier apparaît aussi dans de nombreux ouvrages scientifiques récents consacrés aux manuscrits médiévaux $\mathrm{du} X \mathrm{XV}^{\mathrm{e}}$ siècle grâce à son goût pour les Lettres et les livres qu'il partageait apparemment avec son arrière-petit-fils ${ }^{4}$ et qui leur vaut une place, certes fort modeste, dans l'histoire de l'art pour le premier et dans l'histoire de la littérature pour le second. Ce lien que le cloisonnement des époques et des genres éclipse bien souvent est évidemment à souligner. Même si Louis du Périer est un « important bibliophile lyonnais ${ }^{5}$, il faut néanmoins constater qu'aucune étude ne lui a jamais été consacrée, qu'on ne connaît pratiquement rien de sa biographie et que même la liste complète des manuscrits qu'on peut lui rattacher n'a jamais été faite ${ }^{6}$. Cet article est donc l'occasion de revenir sur ce personnage méconnu de l'histoire lyonnaise mais aussi, plus

\footnotetext{
${ }^{1}$ Pour Raymond Lebègue, « La Consolation à $M$. Du Périer est assurément le plus célèbre des poèmes qui ont paru en France entre les sonnets à Hélène et les Fables de La Fontaine » (Raymond Lebègue, «L'origine de la "Consolation à du Périer" », Comptes rendus des séances de l'Académie des Inscriptions et Belles-Lettres, 86 année, 1942, p. 225).

${ }^{2}$ Marguerite du Périer était la fille de François du Périer, écuyer, premier consul d'Aix en 1593, gentilhomme de la chambre du roi en 1607 et de Catherine Estienne. Elle fut baptisée le 2 février 1593 et mourut en 1598. Ami de son père, François de Malherbe composa peu après une Consolation à du Périer qui fut imprimé pour la première fois en 1607. César de Nostredame, le fils de Michel de Nostredame, écrivit également une ode Sur le trépas de demoiselle Marguerite du Périer, décédée en l'âge de cinq ans.

${ }^{3}$ Les généalogistes tentent de lier Louis du Périer à des homonymes nobles de Provence du XIV siècle puis à la famille homonyme bretonne des comtes de Quintin, attestée depuis le $\mathrm{XI}^{\mathrm{e}}$ siècle.

${ }^{4}$ François du Périer, le père de Marguerite, est en effet l'arrière-petit-fils en ligne directe de Louis du Périer.

${ }^{5}$ François Avril, Nicole Reynaud, Les manuscrits à peintures en France : 1440-1520, Paris, Flammarion, 1995 , p. 201.

${ }^{6}$ Nous avons proposé cette liste dans notre thèse sur les livres à Lyon à la fin du XV siècle (Jean-Benoît Krumenacker, «Du manuscrit à l'imprimé : la révolution du livre à Lyon », Thèse de doctorat de l'université de Lyon, 26 janvier 2019). Nous y reviendrons au cours de cet article.
} 
largement, de présenter un exemple de carrière réussie d'un bourgeois lyonnais de la fin du $\mathrm{XV}^{\mathrm{e}}$ siècle. Ce cursus de Louis du Périer, retracé en grande partie grâce au dépouillement des archives lyonnaises et en particulier des registres de délibération du consulat lyonnais, enrichit l'histoire sociale lyonnaise à la jonction du Moyen Âge et de l'époque moderne en éclairant les pratiques du consulat lyonnais et les liens existant dans une frange de la population de la ville qui exerce le pouvoir et qui est la principale interface de la ville avec la royauté française. Louis du Périer est un exemple remarquable du passage réussi de la bourgeoisie urbaine à la noblesse grâce à des réseaux locaux et nationaux. Rare mais non unique, comme nous le verrons au cours de cet article, ce parcours illustre la position sociale de certaines familles du patriciat lyonnais avant l'institutionnalisation de l'anoblissement des consuls lyonnais et avant une brillante Renaissance lyonnaise qui a attiré bien plus les regards que cette période charnière de la seconde moitié du $X V^{\mathrm{e}}$ siècle qui constitue la fin d'un long Moyen Âge lyonnais ${ }^{7}$.

\section{ÉLEMENTS BIOGRAPHIQUES}

L'origine de Louis du Périer est tout à fait mystérieuse : on ne connaît ni son père, ni sa mère, ni aucun membre de sa famille. En considérant le reste de sa vie, on peut supposer qu'il serait né, sans grande certitude, vers 1440, probablement dans le Lyonnais. La première attestation de son existence est son mariage avec Anne Tèze le 14 juillet $1465^{\circ}$. La famille Tèze $^{9}$ appartient alors à la bourgeoisie lyonnaise mais ne fait pas encore partie de l'élite consulaire. Le premier consul issu de cette famille est Louis Tèze, notaire et drapier, élu au consulat pour la première fois en 1484. Ce Louis Tèze a une carrière dans la vie municipale lyonnaise parfaitement contemporaine de celle de Louis du Périer et on peut supposer qu'il est un frère ou un cousin d'Anne. À une date inconnue, Louis du Périer se remarie avec Marguerite Baronnat, cette dernière étant désignée comme sa veuve dans des documents fiscaux lyonnais ${ }^{10}$. Ce mariage est bien plus prestigieux que le premier. La famille Baronnat est alors l'une des plus importantes familles consulaires lyonnaise, ayant accédé au consulat à partir de 1429 et cumulant 21 mandats entre 1417 et $1519^{11}$. Il est possible que Louis du Périer ait eu une autre épouse entre Anne et Marguerite. L'un des manuscrits que Louis du Périer a fait réaliser vers 1480-1490, celui de La Bouquechardière ${ }^{12}$, avec un décor enluminé à ses armes, possède en effet dans la bordure la répétition des initiales L et I liées par un lacs $\mathrm{d}^{\prime}$ amour rouge ${ }^{13}$. Le L peut correspondre à Louis mais le I ne coïncide ni avec Anne Tèze, ni avec Marguerite Baronnat, ni d'ailleurs avec le patronyme du Périer ou le prénom des enfants

\footnotetext{
${ }^{7}$ Sur Lyon et la société lyonnaise de la fin du Moyen Âge à la Renaissance, outre les histoires générales de la ville de Lyon (par exemple: André Pelletier, Jacques Rossiaud, Françoise Bayard, Pierre Cayez, Histoire de Lyon des origines à nos jours, Lyon, ELAH, 2007), il faut surtout se reporter aux travaux de Jacques Rossiaud et, en particulier, le recueil qui a été fait de ses articles sur Lyon dans : Jacques Rossiaud, Lyon 1250-1550, réalités et imaginaires d'une métropole, Seyssel, Champ Vallon, 2012. On peut également ajouter : Jean-Louis Gaulin et Susanne Rau, Lyon vu/e d'ailleurs, 1245-1800, Lyon, Presses Universitaires de Lyon, 2009).

${ }^{8}$ Fleury Vindry, Les parlementaires français au XVI siècle, Tome I: Parlements d'Aix, Grenoble, Dijon, Chambéry, Dombes, Paris, Librairie Honoré Champion, 1909, p. 32. Nous n'avons pas retrouvé la source de cette information, probablement issues des dossiers bleus de Charles d'Hozier.

${ }^{9}$ Parfois aussi orthographié Tézé, Thèze, Thésé...

${ }^{10}$ Archives municipales de Lyon, CC 39, $\mathrm{f}^{\circ} 10$.

${ }^{11}$ Caroline Fargeix, Les élites lyonnaises du XVe siècle au miroir de leur langage, Paris, De Boccard, 2007, p. 188. Il s'agit de la quatrième famille la plus représentée au consulat.

12 Genève, ms. fr. 70.

${ }^{13} \mathrm{Au}$ folio 3. Ces initiales, peintes en bleues sur le ruban doré de la bordure, sont alternativement $\mathrm{L}$ et I puis I et L.
} 
connus de Louis ${ }^{14}$. Généralement, ce motif d'initiales liées dans le décor d'un manuscrit correspond à celles du commanditaire et de son épouse, ce qui permettrait d'envisager une autre épouse avec cette initiale. L'activité de Louis du Périer au cours des années 1460-1470 est inconnue. D'après les documents rassemblés au XIX ${ }^{\mathrm{e}}$ siècle par Ferdinand Frécon sur les familles consulaires lyonnaises ${ }^{15}$, Louis du Périer est alors commis pour le roi au payement de 400 lances de ses ordonnances puis à la recette des finances des pays albigeois. Cette dernière fonction est attestée dans le colophon d'un manuscrit qu'il fait réaliser au cours des années 1470-1480 ${ }^{16}$. Il s'y intitule « receveur du roi en Albigeois » ${ }^{17}$, c'est-à-dire officier du fisc royal. Néanmoins, ce manuscrit est réalisé à Lyon par un scribe lyonnais et enluminé par un atelier lyonnais ce qui atteste que Louis du Périer est resté lié à cette ville.

\section{LES PREMIERES ANNEES AU SERVICE DE LA VILLE}

À partir de 1484, Louis du Périer apparaît dans la vie politique lyonnaise. Celle-ci peut être retracée en partie grâce aux délibérations municipales. En effet, le consulat reçoit régulièrement des requêtes diverses de Lyonnais, mande des experts pour des questions précises et surtout convoque les notables de la ville, en nombre assez variable allant d'une dizaine à une centaine de personnes selon les séances, pour les affaires les plus importantes pour lesquelles il convient de consulter les Lyonnais. Dans les années 1480-90 en particulier, la question des foires est l'occasion de nombreuses convocations des notables pour décider des initiatives à tenir et rassembler des fonds. Le secrétaire de séance note le nom des personnes présentes ce qui permet d'avoir un panorama des notabilités œuvrant en ville. Le nom de Louis du Périer semble apparaître pour la première fois ${ }^{18}$ dans les délibérations consulaires le 18 mars 1484 (1483 ancien style) ${ }^{19}$. Il y figure en bonne place ${ }^{20}$, la cinquième sur les dix notables convoqués, le second ${ }^{21}$ parmi les «simples » citoyens après Jean Buatier qui a alors déjà accompli six mandats consulaires et est certainement son aîné ${ }^{22}$. Le sujet abordé est apparemment les foires ${ }^{23}$ et Louis du Périer est l'une des cinq personnes à donner son avis d'après le compte-rendu. Dans cette délibération, comme dans les suivantes où il

\footnotetext{
14 Hippolyte Aubert propose éventuellement d'y voir un J pour Jaspard (Gaspard), le fils aîné de Louis (Hippolyte Aubert, Notices sur les manuscrits Petau conservés à la bibliothèque de Genève, Bibliothèque de l'école des chartes, 1909, tome 70, p. 485). Mais les sources lyonnaises orthographient très majoritairement ce prénom avec un $\mathrm{G}$ et sinon avec un $\mathrm{C}$ (Caspard), ce qui correspond avec la valeur de la consonne initiale, prononcée certainement d'un son proche du [k].

${ }^{15}$ Archives départementales du Rhône, 106J. Il s'agit de travaux manuscrits restés inédits et malheureusement sans mention des sources. Le volume dans lequel on trouve mention de la famille du Périer est coté 106J11 (familles P-Q). Un certain nombre d'informations familiales, reprises de généalogies plus anciennes, sont erronées.

${ }^{16}$ Chantilly, Musée Condé, ms 833-834.

17 «Se present livre de Vallere le Grant est a noble homme Loys du Perier recepveur pour le roy nostre sire ou païs d'Albyjoys ».

18 Nous avons remonté l'intégralité des délibérations consulaires jusqu'à la fin des années 1470 puis fait quelques sondages pour les années antérieures sans trouver de mention de Louis du Périer. Néanmoins, il existe aussi des lacunes dans la série des délibérations.

19 Archives municipales de Lyon (AML), BB17. Minutes sans foliotation et dans un ordre plus ou moins chronologique.

${ }^{20}$ Les noms des présents sont presque toujours donnés selon un ordre hiérarchique avec tout d'abord les officiers du roi et de l'archevêque, les docteurs et licenciés en droit puis les autres Lyonnais selon un ordre de préséance réfléchi. C'est apparemment le cas ici.

${ }^{21}$ Les trois premiers personnages cités sont les officiers et les gradués.

${ }^{22}$ Il en accomplira en tout huit. Après eux suivent Louis Tèze, Philippe [?], Guillaume Bellièvre, Claude Grant et Catherin [?].

${ }^{23}$ Seules des minutes très difficilement lisibles ont été conservées pour cette époque.
} 
apparait, il n'est jamais désigné comme receveur du roi alors que le secrétaire donne volontiers les offices royaux des présents, ce qui pourrait signifier qu'il ne l'est alors plus. Le lendemain, le 19 mars $^{24}$, les conseillers et notables, parmi lesquels se trouve à nouveau Louis du Périer, décident d'envoyer des émissaires aux états de Languedoc pour le fait des foires. Humbert de Varey ${ }^{25}$ propose le nom de Louis du Périer et celui-ci accepte de partir pour les affaires de la ville. La question des foires est alors une question brûlante pour Lyon ${ }^{26}$. La ville avait obtenu des foires en 1444 tout d'abord deux puis trois et quatre en 1463. Les foires se développent surtout au cours des années 1460 quand elles supplantent celles de Genève. L'ensemble des banques italiennes s'installent alors à Lyon qui devient l'une des places centrales du commerce européen. Mais les autres villes françaises se sentent désavantagées et accusent les foires de Lyon de faire sortir l'or du royaume. Les États généraux de Tours prennent en compte ces plaintes et Charles VIII abolit les foires de Lyon le 8 mars 1484. La bourgeoisie lyonnaise va alors multiplier les initiatives et les cadeaux pour récupérer les foires. La ville se voit restituer deux foires en 1489 puis les quatre d'origine sont rétablies en 1494 et ne seront plus contestées.

Le départ de Louis du Périer n'est pas immédiat puisqu'on le retrouve présent à des assemblées de la ville jusqu'au premier avril. Il disparaît ensuite et le 12 juin $1484^{27}$ le consulat mande les notables de la ville pour lire «trois paires de lettres missives envoyées [à eux] par Louis du Périer étant à Montpellier ${ }^{28}$ » concernant l'appel fait par la ville de la suppression des foires. Du Périer leur apprend que l'appel a été reçu par un notaire d'Aubenas, Vidal Sabatier, et les prévient des « abus, torts, injures et molestations » dont il a été victime. Le 14 juin $^{29}$, consuls et notables sont convoqués pour entendre une autre lettre de du Périer. Celui-ci est alors emprisonné à Montpellier et signale que les Montpelliérains ont envoyé un émissaire en Bourgogne pour informer du déportement des foires. Les Lyonnais décident alors d'envoyer leur propre émissaire en Bourgogne pour s'opposer à l'annonce du déportement des foires et en faire appel ainsi qu'un homme d'importance à Montpellier pour « consoler » et « soulager» du Périer et passer à Aubenas pour avoir les pièces de l'appel. Ces malheurs ne durèrent guère puisque Louis du Périer est à nouveau présent à Lyon pour une séance du consulat le 11 juillet $1484^{30}$. Il semble ensuite disparaitre pendant l'été avant d'être à nouveau présent à des séances du consulat à l'automne. Les péripéties de son voyage à Montpellier sont connues un peu plus en détail grâce à une délibération du 23 décembre 1484, conservée en deux exemplaires avec des détails légèrement différents ${ }^{31}$. Ce jour-là, Louis du Périer se présente au consulat à propos du payement de deux voyages fait par lui pour le compte de la ville, l'un à la cour en France ${ }^{32}$ auprès du roi, l'autre à Montpellier aux États de Languedoc. Nous n'avons pas trouvé de trace du premier voyage mais la ville envoie régulièrement à cette époque plusieurs consuls et notables auprès du roi pour intervenir en sa

\footnotetext{
${ }^{24}$ AML, BB17.

25 «Monseigneur le Maistre » désigne en effet Humbert de Varey dans les archives lyonnaises, ce dernier étant alors maître d'hôtel du roi.

${ }^{26}$ Sur la question des foires de Lyon au XV siècle, voir : Marc Brésard, Les foires de Lyon aux XVe et XVI siècles, Paris, Auguste Picard, 1914.

${ }^{27}$ AML, BB15, f $228 v^{\circ}$.

${ }^{28}$ Les États du Languedoc y sont alors rassemblés.

${ }^{29}$ AML, BB15, f $228 \mathrm{v}^{\circ}-229 \mathrm{r}^{\circ}$.

${ }^{30} \mathrm{AML}, \mathrm{BB} 15, \mathrm{f}^{\circ} 232 \mathrm{v}^{\circ}$ sqq.

${ }^{31}$ AML, BB15, f $\mathrm{f}^{\circ} 276 \mathrm{r}^{\circ}-\mathrm{v}^{\circ}$ et BB18, $\mathrm{f}^{\circ} 2 \mathrm{v}^{\circ}-3 \mathrm{r}^{\circ}$.

${ }^{32}$ La France, comme région, correspond à peu près à l'actuelle Île-de-France. On peut supposer que le roi et sa cour n'étaient alors pas à Paris.
} 
faveur. Louis du Périer déclare ne pas se contenter du taux des jours de vacations qu'on lui a fait, à savoir 30 sols tournois par jour de voyage, mais demande autant que Jean Baronnat ${ }^{33}$ et d'autres. Il présente ses travaux et expose les « empêchements qu'il a eu au [cours de son voyage] en Languedoc où il fut emprisonné et molesté par le président Lauret $^{34}$ » : son bagage pris, ouvert et inventorié, ses chevaux arrêtés, lui-même condamné à 25 livres tournois d'amende par le président Lauret pour avoir «troublé les états» et «plusieurs autres vitupéries, torts, griefs et oppressions ». Même après la libération de prison de Louis du Périer, Lauret « fait arrêter et détenir plusieurs des lettres et autres choses que ledit du Périer avait en Languedoc ». Finalement, le consulat n'accepte pas l'augmentation demandée ${ }^{35}$ mais offre à Louis du Périer 25 livres tournois en récompense de ses efforts et 25 autres livres tournois en dédommagement de ses frais en plus du tarif habituel. Les consuls ordonnent donc de verser à Louis du Périer pour l'ensemble de ses frais la somme de 108 livres 7 sols et 6 deniers tournois ${ }^{36}$.

La mission de du Périer à Montpellier semble avoir été particulièrement houleuse avec un affrontement direct avec le président Lauret mais également avec les États de Languedoc devant qui du Périer paraît avoir fait scandale. Si le conflit entre Lyon et les villes du sud est particulièrement vif à cette époque du fait des foires que chacune veut récupérer pour amener à elle les richesses et les flux de marchandises, cet épisode de confrontation directe, alors même que Lyon cherche à tout prix à récupérer ses foires, en cristallise la violence. Cette mission a apparemment été un échec : les affaires lyonnaises n'ont pas progressé et aucun des appels de la suppression des foires n'aura de conséquence rapide. Du Périer qui devait certainement tenter de trouver des appuis en Languedoc ou des arrangements avec les villes concurrentes de Lyon n'a pas été écouté et a fini dans les geôles montpelliéraines. Les Lyonnais ne semblent pas lui en tenir rigueur. Le consulat l'indemnise et le récompense, lui confie ensuite d'autres missions et un peu plus d'un an plus tard, en décembre 1485, il est élu consul pour les années 1486 et 1487.

L'année 1485 ne voit aucune action particulièrement remarquable de Louis du Périer. Il est présent à une dizaine d'assemblées des notables tout au long de l'année et ne semble absent qu'à l'une d'elles. Il fait alors clairement partie de la vingtaine de citoyens lyonnais qui assistent les douze consuls dans le gouvernement de la ville. Enfin, le 15 décembre 1485, les maîtres des métiers choisissent de l'élire au consulat pour les deux ans à venir avec Pierre Brunier, Étienne Laurencin, Étienne Garnier, Jean Caille et François de Genas ${ }^{37}$. Le consulat

\footnotetext{
${ }^{33}$ Jean Baronnat est consul en 1483 en remplacement de Pierre Thomassin élu en 1482. À ce titre, il était peutêtre mieux indemnisé pour ses voyages. Le taux demandé par Louis du Périer n'est pas explicité.

${ }^{34}$ Bernard Lauret, premier président du parlement de Toulouse de 1472 à 1495.

${ }^{35} \mathrm{La}$ raison évoquée est d'éviter des conséquences dommageables. Probablement le consulat ne veut-il pas banaliser ce type de demande et décide de s'en tenir sans exception au tarif fixé.

${ }^{36}$ AML, CC514, pièce 16 (23 décembre 1484). L'ordonnance de payement adressée au trésorier est conservée dans les archives comptables de la ville. Elle détaille les frais occasionnés par le voyage de Louis du Périer, qui montent à 148 livres, 7 sols et 6 deniers tournois desquels il faut déduire un acompte de 40 livres tournois reçu par du Périer à son départ. Son voyage a duré 63 jours entiers et on lui verse donc 119 livres 10 sols pour son voyage, ses peines et travaux (ce qui correspond à 63 jours à 30 sols [=94 livres, 10 sols] plus 25 livres tournois), 25 livres tournois pour le remboursement de son amende et 3 livres 17 sols et 6 deniers tournois pour un payement à maître Vidal Sabatier pour l'appel des foires.

${ }^{37}$ AML, BB15, f $341 v^{\circ}$.
} 
lyonnais est alors constitué de douze membres élus par six chaque année pour deux ans ${ }^{38}$. Ils sont élus courant décembre par les maîtres des métiers eux-mêmes nommés par le consulat quelques jours plus tôt, ce qui permet aux principales familles consulaires de contrôler le système. L'entrée effective en fonction n'est pas très claire et on voit arriver les nouveaux conseillers et disparaître les anciens des séances du consulat au cours du printemps, voire de l'été qui suivent. Les séances du consulat n'ont pas de régularité précise et se tiennent selon les nécessités. En moyenne, on a cependant environ deux rencontres par semaine ${ }^{39}$. Du fait de la fréquence de ces réunions, les consuls sont rarement tous présents et certains affichent même des taux d'absentéisme impressionnants ${ }^{40}$. L'inscription des noms des présents au début des délibérations de chaque séance permet d'avoir une connaissance assez fine de l'attention portée à la ville par chaque consul et de leur présence à Lyon $^{41}$.

Au cours de l'année 1486, Louis du Périer ne semble intégrer vraiment le consulat qu'à l'été. Il est convoqué pour prêter serment le 18 juillet ${ }^{42}$ mais n'est compté parmi les consuls de la ville qu'à partir de la séance du 22 août ${ }^{43}$. Il participe néanmoins entre temps à la vie de la cité puisqu'on le retrouve avec les notables dans des assemblées du début de l'année, du printemps et de l'été 1486. Le mercredi 23 août 1486, le lendemain même de l'apparition de du Périer au consulat, celui-ci décide «d'envoyer devers le roy notre sire quelque personnage saige et de bonne apparence ${ }^{44}$ pour diverses affaires dont les foires et un conflit avec les habitants du plat pays de Lyonnais. Les consuls élisent Louis du Périer pour cette tâche et ce dernier accepte la mission et se déclare prêt à partir sous huit jours. Le lendemain, le 24 août, devant l'assemblée des notables qui demande un départ rapide, Louis du Périer réitère son accord de partir, d'ici trois matins cette fois-ci, mais demande à être accompagné d'un autre Lyonnais d'importance et fait remarquer qu'il sert de cette façon la ville au détriment de ses propres affaires. Le mardi 29 août, Louis du Périer reçoit une avance de 80 livres tournois pour les frais du voyage et son compagnon, François Buclet, docteur en lois, une avance de 120 livres tournois ${ }^{45}$. Les consuls leur demandent de partir le lundi qui suit pour la cour avec des recommandations sur les soutiens à chercher ainsi qu'un crédit de 10000 francs pour la réintégration des foires. Cette mission est encore abordée dans les délibérations des jours qui suivent. Ainsi le dimanche 3 septembre, Buclet et du Périer reçoivent le mandat de la ville d'engager 10000 francs pour les foires et 2000 pour les affaires du plat pays de Lyonnais ; puis le lundi 4 septembre, on leur recommande de passer à Pont-d'Ain en Bresse pour voir le cardinal de Bourbon, archevêque de Lyon, « pour savoir s'il lui plaira leur rien commander et lui supplier qu'il lui plaise écrire deux ou trois lignes de sa

\footnotetext{
${ }^{38}$ Ce système est en usage depuis 1447. Auparavant, les douze consuls étaient élus ensemble pour un an. Mais le problème de la continuité entre deux années a conduit le consulat à ne plus renouveler que la moitié de ses effectifs chaque année et donc d'allonger à deux ans la durée des mandats.

${ }^{39}$ Caroline Fargeix, Les élites lyonnaises, op. cit. (n. 11), p. 284.

${ }^{40}$ Celui-ci atteint même parfois chez certains conseillers les 75-80\% ! En moyenne, sept à huit consuls sont présents aux séances, soit un taux d'absence moyen d'environ $40 \%$ : Caroline Fargeix, Les élites lyonnaises, op. cit. (n. 11), p. 288-290.

${ }^{41} \mathrm{Si}$ la présence indique forcément que l'intéressé est à Lyon, l'absence apporte moins d'informations tant les raisons sont nombreuses de manquer une séance du consulat. Une absence prolongée sur plusieurs semaines peut, en revanche, laisser supposer un voyage loin de Lyon.

42 AML, BB15, f $366 \mathrm{v}^{\circ}$.

${ }^{43}$ D'après BB19. BB15 donne, probablement à tort, à la place et juste pour cette séance Louis Tèze.

${ }^{44}$ AML, BB15, $\mathrm{f}^{\circ} 371 \mathrm{r}^{\circ}$.

${ }^{45}$ La différence de somme n'est pas expliquée. Buclet comme du Périer sont alors consuls mais seul le premier a un grade universitaire qui justifie peut-être un salaire plus important.
} 
main à monseigneur le connétable ${ }^{46}$ pour les aider à avoir la réintégration des foires ${ }^{47}$. Du Périer et Buclet assistent encore à une séance le mardi 5 septembre puis disparaissent des délibérations lyonnaises à partir du 7.

Cette ambassade auprès de la cour dure quelques temps. Le 14 novembre, le consulat envoie de nouvelles affaires à Paris à François Buclet et Louis du Périer. Buclet revient à Lyon fin novembre et fait son rapport au consulat le 28 novembre ${ }^{48}$, tandis que du Périer reste auprès du roi. Le 29 décembre ${ }^{49}$, les consuls lisent une lettre envoyée par Louis du Périer et lui demandent en retour de rester à la cour jusqu'à la fin des affaires qu'il suit. Ce même jour, un chevaucheur est payé pour avoir porté à du Périer courant décembre des mémoires sur différentes affaires ${ }^{50}$. Le 6 janvier 1487, le consulat lit une autre lettre de du Périer, apporté par un de ses serviteurs, et lui répond à nouveau de rester en cour jusqu'à la fin des affaires dont il a la charge. On peut supposer que la réponse du 29 décembre apporté par les messagers de la ville à du Périer et la lettre lue le 6 janvier et portée par un serviteur de du Périer se sont croisés en chemin. Il est également probable que Louis du Périer demande dans ses courriers à pouvoir rentrer lui aussi. Le 23 mars $1487^{51}$, le consulat donne une nouvelle procuration à François Buclet et à Louis du Périer, alors toujours absent, pour la somme de 5000 francs à laquelle s'ajoute une seconde de 3000 francs au cas où la première somme ne suffise pas, toujours pour la réintégration des foires. Buclet repart peu de temps après avec ces procurations pour rejoindre Louis du Périer à la cour. Le 28 mars $1487^{52}$, François Buclet reçoit 200 livres tournois pour son voyage et cent livres tournois qu'il doit porter à Louis du Périer comme avance sur ses remboursements de frais. Les délibérations municipales connaissent à cet endroit quelques lacunes mais on trouve le 10 avril 1487 une nouvelle dépense pour un messager envoyé à la cour porter des lettres de la ville à du Périer. Enfin, le retour de ce dernier s'effectue avant le 12 mai 1487, environ huit mois après son départ. Ce jour-lă ${ }^{53}$, il fait le rapport de ses activités à la cour devant les autres consuls.

Par la suite, du Périer peut enfin occuper ses fonctions de consul dont il n'a guère eu le temps de profiter avant son départ. Sur environ 70 séances du consulat tenues entre son retour et la fin de l'année 1487 , son taux de présence est de $72 \%$, ce qui est plus que correct. Outre des absences très ponctuelles, il n'assiste à aucune séance entre le 18 septembre et le 31 octobre 1487, ce qui peut correspondre à un voyage hors de Lyon. Parmi les péripéties consulaires de l'année 1487 , on peut noter le 30 juillet 1487 une délibération unique ${ }^{54}$ par laquelle les consuls désignent quatre d'entre eux pour expédier certaines affaires courantes de la ville. Dix conseillers sont présents et chacun donne quatre noms, personne ne votant pour lui-même. Le résultat des votes ${ }^{55}$ permet de dessiner plusieurs groupes : Le Maistre, Garnier, du Rieux, Taillemond et du Périer récoltent entre 5 et 9 voix, c'est-à-dire sont le choix de la

\footnotetext{
${ }^{46}$ Le connétable de France est alors Jean II de Bourbon, duc de Bourbon et frère de l'archevêque de Lyon.

${ }^{47}$ AML, BB15, $\mathrm{f}^{\circ} 374 \mathrm{v}^{\circ}$.

${ }^{48}$ AML, BB19, fo $30 v^{\circ}$.

${ }^{49}$ AML, BB19, fo $33 \mathrm{r}^{\circ}$.

${ }^{50} \mathrm{On}$ y apprend au passage qu'il lui a fallu 18 jours entiers pour faire l'aller-retour entre Lyon et la cour.

${ }^{51}$ AML, BB19, $\mathrm{f}^{\circ} 41 \mathrm{v}^{\circ}$.

${ }^{52}$ AML, BB19, $\mathrm{f}^{\circ} 41 \mathrm{v}^{\circ}$.

${ }^{53}$ AML, BB19, $\mathrm{f}^{\circ} 42 \mathrm{r}^{\circ}$. Ce rapport n'est pas explicité.

${ }^{54}$ Pour la période 1417-1519, ce scrutin pour lequel on a le détail des votes est unique : Caroline Fargeix, Les élites lyonnaises, op. cit. (n. 11), p. 317-319; AML, BB19, f ${ }^{\circ} 53 \mathrm{r}^{\circ}$.

55 Sur 9 voix possibles (10 présents, aucun ne votant pour lui) : Le Maistre 9 voix, Garnier 8, du Rieux 7, Taillemond 6, du Périer 5, Rousselet 3, Laurencin 1, Baronnat 1. Rousselet et Caille sont absents ce jour-là.
} 
majorité des conseillers ; Rousselet, Laurencin et Baronnat ont quelques voix isolées ; les quatre derniers - Caille, Brunier, Genas et Colombier - n'en ont aucune. Finalement, avec cinq voix, Louis du Périer n'est pas nommé mais il fait clairement partie du groupe le plus influent du consulat même s'il en est aussi le plus inexpérimenté ${ }^{56}$. Caroline Fargeix utilise ce vote pour montrer des dissensions qui peuvent exister entre les conseillers avec, par exemple, Taillemond et Rieux qui ne votent pas l'un pour l'autre ${ }^{57}$. Si l'on regarde plus spécifiquement du Périer, on peut voir qu'il vote pour les quatre autres principaux conseillers (Le Maistre, Garnier, du Rieux et Taillemond) et que ces derniers votent également pour lui à l'exception de Garnier. Peu après, on peut aussi mentionner que du Périer est envoyé début août ${ }^{58}$ en ambassade auprès du cardinal de Bourbon dans son hôtel lyonnais pour régler, sans succès, un différend entre le consulat et l'archevêque de Lyon à propos d'une représentation d'un mystère sur la Passion. Enfin, le 3 décembre 1487, au cours de l'élection des maîtres des métiers, Louis du Périer est nommé représentant des «terriers » pour l'année 1488. Les terriers, c'est-à-dire les rentiers propriétaires terriens, forment le premier métier dans l'ordre protocolaire des métiers représentés dans les instances municipales lyonnaises. En 1488, du Périer reste présent aux séances du consulat jusqu'au mois d'août mais de façon moins assidue puisque son taux de présence n'est plus que de $58 \%$. En janvier $1488^{59}$, il est envoyé à Tournon rencontrer monseigneur de Tournon toujours pour l'affaire des foires et en est payé le 21 juin. Les cinq jours de voyage lui sont payés dix livres tournois ${ }^{60}$. Après son départ du consulat et au cours de l'année 1489, on le retrouve régulièrement parmi les notables convoqués en assemblée par les consuls.

\section{UN NOTABLE LYONNAIS BIEN INSTALLE}

Le 31 avril $1489^{61}$, le consulat lit des lettres du procureur de la ville auprès du roi qui recommande d'envoyer rapidement « un ou deux des plus gens de bien et entendus de ladite ville» pour comparaître devant le roi ou ses commissaires dans une affaire de grande importance de division des tailles avec le plat pays que la ville semble en train de perdre. Les notables de la ville élisent pour cette tâche François Buclet et Claude Le Charron, docteurs en lois, et Louis du Périer, bourgeois. Ces trois personnages sont convoqués le lendemain par le consulat pour savoir si deux d'entre eux veulent bien accepter cette mission. Louis du Périer, déjà présent la veille, s'excuse en prétextant un voyage qu'il doit nécessairement faire en Provence $^{62}$. De fait, Louis du Périer assiste à une assemblée le 14 mai puis disparaît

\footnotetext{
${ }^{56}$ Du fait de son voyage à Paris, son expérience concrète des affaires courantes du consulat est de trois mois. Le Maistre en est à son quatrième mandat, Garnier et Taillemont à leur deuxième, du Rieux en est également à son premier mandat mais il est docteur en droit. Or la présence des juristes devient alors indispensable au consulat.

${ }^{57}$ Caroline Fargeix, Les élites lyonnaises, op. cit. (n. 11), p. 318. On peut supposer que les votes Laurencin, Baronnat et probablement Rousselet sont plus des votes contre certains membres du groupe principal, entre qui l'élection se joue, que vraiment pour ces consuls. Si l'on interprète ces votes en ce sens, on a 2 rejets de du Périer, du Rieux et Taillemond et un rejet de Garnier. Pour du Périer, c'est Garnier et Baronnat qui préfèrent voter pour un petit candidat que pour lui. Concernant Baronnat, la situation peut surprendre dans la mesure où du Périer épouse une Baronnat. Mais la date de ce mariage est inconnue et on peut aussi supposer que, comme aucun consul ne vote pour lui-même, on s'abstienne aussi de nommer des proches.

${ }^{58}$ AML, BB19, $\mathrm{f}^{\circ} 53 \mathrm{v}^{\circ}-54 \mathrm{r}^{\circ}$. L'affaire est évoquée dans : Tania Levy, Les peintres de Lyon autour de 1500 , Rennes, Presses universitaires de Rennes, 2017, p. 154.

${ }^{59}$ AML, BB19, $\mathrm{f}^{\circ} 82 \mathrm{v}^{\circ}$.

${ }^{60}$ On peut noter que le taux de remboursement est supérieur à celui qu'il avait pour de son voyage à Montpellier (30 sols tournois par jour) alors qu'il n'était pas encore consul.

${ }^{61}$ AML, BB19, $\mathrm{f}^{\circ} 130 \mathrm{v}^{\circ}-131 \mathrm{r}^{\circ}$.

${ }^{62}$ AML, BB19, f $131 \mathrm{v}^{\circ}$.
} 
complètement des registres municipaux jusqu'au 2 novembre 1489. La mention de ce voyage en Provence est extrêmement importante. En effet, la qualification principale de Louis du Périer dans les documents $\mathrm{du} \mathrm{XV}^{\mathrm{e}}$ siècle jusqu'à aujourd'hui est son office de visiteur des gabelles à sel de Provence. On indique généralement qu'il reçoit cet office par ordonnance royale de Charles VIII donnée à Amboise le 4 décembre $1486^{63}$. Or, dans l'abondante documentation municipale lyonnaise, Louis du Périer n'est mentionné comme « visiteur du sel en Provence pour le roi nostre sire » qu'à partir du 2 novembre 1489, c'est-à-dire à son retour de ce voyage en Provence qui l'empêche de partir à Paris pour la ville. On peut donc supposer que c'est seulement au cours de cette année 1489 qu'il prend réellement la charge de visiteur du sel en se rendant en Provence. Ce serait cette date et non 1486 qu'il faudrait prendre comme terminus post quem ou ante quem des mentions où ce titre est absent ou présent. C'est avec ce titre de visiteur qu'il est par la suite mentionné jusqu'à sa mort dans les archives municipales lyonnaises ${ }^{64}$ et qu'il est connu aujourd'hui. Cet office royal lui assure une certaine stature à Lyon. Il compte alors parmi les officiers royaux, toujours nommés à part dans les listes de notables et reçoit le prédicat de «Monseigneur le visiteur » qui le distingue du commun.

En 1490, du Périer est régulièrement présent dans les assemblées des notables et le 12 décembre $1490^{65}$, il est élu pour un second mandat avec François Buclet, Pierre Brunier, Jean Rousselet, Étienne Laurencin et Jean Le Maistre pour 1491-1492. Nous n'avons pas trouvé trace de son serment mais il apparaît aux séances du consulat à partir de juin. Son taux de présence en 1491, après juin, est assez médiocre, aux environs de 47\%. Auparavant, le 31 janvier $1491^{66}$, Louis du Périer recevait 100 livres tournois de la ville pour les services de son serviteur François qui était allé à la cour avec les envoyés de la ville François Buclet, Antoine de Villars et Pierre Le Maistre pendant sept mois et demi ${ }^{67}$. L'année 1492 est marquée par une implication plus forte de du Périer dans le consulat. Son taux de présence remonte à $77 \%$. Surtout, il apparait dans la liste des consuls en seconde position après Pierre Palmier, visiteur du sel en Lyonnais, et préside les séances du consulat en l'absence de ce dernier. Le $1^{\mathrm{er}}$ mai 1492, il est également désigné comme lieutenant du capitaine de la ville pour le côté de l'empire $^{68}$ en l'absence de Claude Thomassin alors que de nombreuses personnes sont attendues en ville tant pour les foires que pour un pas d'armes ${ }^{69}$. La semaine suivante, le 8 mai 1492, Louis du Périer prête 60 livres tournois à la ville afin de pouvoir salarier Jean Vidille, notaire royal pour qu'il fasse un voyage pour les affaires de la ville, le trésorier de la ville ayant déclaré qu'il n'y avait plus un denier dans les caisses de la municipalité ${ }^{70}$. Il

\footnotetext{
${ }^{63}$ Il faut évidemment noter qu'à cette date, Louis du Périer se trouve à la cour pour les affaires de la ville depuis trois mois. Il aurait donc attendu deux ans après son retour de la cour pour prendre réellement possession de sa charge.

${ }^{64}$ Suivant les comptes-rendus de délibérations, il est appelé « monseigneur le visiteur », « le visiteur du Périer » ou simplement « du Périer ».

${ }^{65}$ AML, BB19, fo $204 \mathrm{v}^{\circ}$.

${ }^{66}$ AML, BB19, fo $209 \mathrm{r}^{\circ}$.

${ }^{67}$ Cela représente une indemnité d'environ 9 sols tournois par jour à du Périer pour le prêt de son serviteur.

${ }^{68}$ La ville de Lyon, située alors sur la Saône, est traditionnellement divisée en un côté de l'empire (rive gauche de la Saône) et un côté du royaume (rive droite).

${ }^{69} \mathrm{AML}, \mathrm{BB} 19, \mathrm{f}^{\circ} 259 \mathrm{v}^{\circ}$. Claude Guerrier est nommé pour le côté du royaume.

${ }^{70} \mathrm{AML}, \mathrm{B} 19, \mathrm{f}^{\circ} 259 \mathrm{v}^{\circ}-260 \mathrm{r}^{\circ}$. «Ledit du Périer voyant et coignoissant ledit voyage estre neccessaire à ladite ville [...] a offert esdits conseillers prester de ses deniers oultre les autres prestz par luy ja faitz et somme de deniers a luy deues de par ladite ville la somme de soixante livres tournois pourveu quil soit rasseure de son remboursement dedans la fin de ce troysieme quartier de ceste présente annee ».
} 
demande à être remboursé avant la fin du troisième trimestre de l'année et déclare être déjà créditeur de la ville, tant par des prêts déjà concédé que par d'autres sommes à lui dues.

En 1493, Louis du Périer reste au consulat jusqu'à la fin du mois de juillet. Son taux de présence aux séances est de $78 \%$ (et même de $89 \%$ jusqu'à la fin du mois de mai). Palmier ne réapparaissant plus au consulat après décembre 1492, du Périer préside toutes les séances du consulat auxquelles il est présent jusqu'à l'apparition, le 17 mars 1493, de Jean Caille, docteur en loi, élu consul trois mois auparavant. En l'absence de ce dernier, Louis du Périer continue de prendre la présidence du consulat jusqu'à son départ. En juin et juillet, il ne semble d'ailleurs présent au consulat quasiment qu'en l'absence de Jean Caille. Lors d'une des dernières séances qu'il préside, le 16 juin $1493^{71}$, Louis du Périer en profite pour demander le soutien officiel de la ville en faveur de son fils, Gaspard du Périer, afin d'obtenir la charge de juge ordinaire de la ville. Il demande que le consulat écrive en ce sens à l'archevêque de Lyon, qui possède alors la justice sur la ville et nomme aux offices de la cour séculière, et indique que son fils est âgé de 25 ans et est docteur dans les deux droits de l'université de Pise ${ }^{72}$, ce qui le rend apte à occuper cet office. Mais la femme de François Buclet fait la même demande par lettre pour son époux ${ }^{73}$ et les consuls préfèrent ne soutenir aucun des deux protagonistes, François Buclet et Louis du Périer ayant «bien servy ladite ville chacun en son endroit ». Finalement, Gaspard du Périer ne semble pas avoir été le choix de l'archevêque.

Contrairement à son fils, Louis du Périer a servi l'archevêque à cette époque. Dans la Nommée de 1493, il apparaît en effet comme « bourgeois, visiteur du sel, courrier de lion ${ }^{74}$. Le courrier ${ }^{75}$, fonctionnaire de robe courte, est le principal officier de la cour séculière, nommé directement par l'archevêque. Il est le bailli de celui-ci sur toutes ses terres, en charge de la justice civile et criminelle à Lyon, du maintien de l'ordre, de la police et du ravitaillement. Les titulaires de cet office ne sont pas des juristes mais des nobles puis, à partir de la fin du XIV ${ }^{\mathrm{e}}$ siècle, des patriciens aux frontières de la noblesse. La charge de juge ordinaire, convoitée par Gaspard, est une lieutenance de celle de courrier avec délégation de la juridiction civile pour laquelle le courrier se repose entièrement sur le juge. Malheureusement, les archives de la juridiction temporelle de l'archevêque ont presque entièrement disparu ${ }^{76}$ et on ne peut pas dire grand-chose sur l'exercice de cette charge par Louis du Périer. En étudiant les hommes de loi lyonnais, René Fédou a reconstitué une liste très lacunaire des titulaires de l'office de courrier ${ }^{77}$ mais on n'a souvent guère plus d'une mention pour chacun d'eux. C'est le cas pour du Périer pour lequel il n'existe que cette unique pièce où l'on détaille plusieurs de ces charges. La documentation municipale ne donne en effet généralement qu'un seul titre par personne et, pour du Périer, c'est toujours « visiteur du sel».

\footnotetext{
${ }^{71}$ AML, BB20, f $95 \mathrm{v}^{\circ}$.

${ }^{72}$ Le choix de cette université est rare pour les Lyonnais. Outre Gaspard du Périer, on ne connaît qu'Humbert de Grôlée qui y ait étudié au XV $\mathrm{XV}^{\mathrm{e}}$ siècle. Turin, Pavie et Ferrare sont les universités italiennes les plus fréquentées alors par les Lyonnais: René Fédou, Les hommes de loi lyonnais à la fin du Moyen Âge, Paris, Les Belles Lettres, 1964, p. 306.

${ }^{73}$ Son mari est alors en déplacement à Grenoble.

74 AML, CC11.

${ }^{75}$ René Fédou, Les hommes de loi lyonnais, op. cit. (n. 72), p. 77-78.

${ }^{76}$ Archives départementales du Rhône (ADR), 10G1455-1457 pour les années 1317-1713. Il n'existe que deux actes conservés entre 1493 et 1504, aucun ne mentionnant le courrier.

${ }^{77}$ René Fédou, Les hommes de loi lyonnais, op. cit. (n. 72), p. 83.
} 
Le service de l'archevêque, pas plus que celui du roi, n'est incompatible avec les activités municipales. Au contraire, la présence d'officiers du roi ou de l'archevêque au consulat facilite la coopération entre les différentes juridictions. Cet office donne un poids particulier à Louis du Périer dans la vie politique lyonnaise. Avant Louis, Benoît du Rieux est attesté comme courrier de Lyon vers $1492-1493$ et l'est encore le 8 mai $1493^{78}$. Les offices de courrier et de juge semblent avoir été pourvus à des dates rapprochées, voire simultanément, et ont été convoités par Louis du Périer et son fils. Il serait évidemment intéressant d'avoir une chronologie plus fine pour savoir si Louis du Périer est déjà courrier quand il soutient son fils pour devenir juge et donc son propre collaborateur. Le fait que les deux principaux offices de la cour séculière soient vacants à peu près en même temps peut laisser supposer une dépendance forte de l'un à l'autre ou que Benoît du Rieux cumulait les deux. Ce cumul est attesté par Fédou mais seulement au début du XIV ${ }^{\mathrm{e}}$ siècle $^{79}$. Néanmoins, le profil de Benoît du Rieux qui est juriste, docteur en droit et qui est donné comme lieutenant du courrier avant d'être courrier pourrait correspondre à un tel cumul. On peut également se demander pourquoi Louis du Périer demande l'appui du consulat pour son fils mais pas pour lui-même. Mais les deux offices, l'un pour les nobles ou les prétendants à cet état, l'autre pour les juristes sont assez différents et la concurrence n'est probablement pas la même, de même que les critères de choix. Si les candidatures de Louis et Gaspard sont à peu près simultanées, on peut supposer que la réussite de Louis a entamé les chances de Gaspard : nommer le père et son fils aux deux principaux offices de la cour séculière pouvant paraître un choix discutable. Louis du Périer semble s'être démis de cette charge avant $1500^{80}$. Claude Guerrier est ensuite attesté comme courrier vers 1504.

Cette même année 1493 meurt Humbert de Varey ${ }^{81}$, le chef de la plus illustre famille consulaire lyonnaise du XV $\mathrm{XV}^{\mathrm{e}}$ siècle $^{82}$. Riche propriétaire terrien, consul en 1459-1460, trésorier de l'archevêque en 1463, élu du Lyonnais de 1462 à 1471, il est anobli en 1473, devient seigneur de Belmont et maître d'hôtel du roi. Il avait épousé Antoinette du Terrail, d'une vieille famille noble du Dauphiné et tante du fameux chevalier Bayard, puis Éléonore de La Ratta, issue de la noblesse italienne. Dans son testament, daté du 31 mai $1491^{83}$, il institue Louis du Périer comme tuteur de son fils et héritier Antoine de Varey alors encore mineur (impuberis) ainsi que comme exécuteur testamentaire de ses volontés, à chaque fois avec Éléonore de La Ratta son épouse. Louis du Périer ne fait pas partie des légataires de Humbert de Varey qui distribue divers dons à des membres de sa famille et de son personnel mais est désigné comme son « compatriote très cher ${ }^{84}$. On peut aussi noter que Humbert de Varey ne désigne comme nobilis vir/mulier que lui-même, son épouse Éléonore, son fils Antoine de Varey, Louis du Périer et un témoin désigné comme écuyer de Saint-Symphorien d'Ozon ${ }^{85}$.

\footnotetext{
${ }^{78}$ Caroline Fargeix, Les élites lyonnaises, op. cit. (n. 11), p. 596 ; René Fédou, Les hommes de loi lyonnais, op. cit. (n. 72), p. 83. Il semble disparaître après cette date.

${ }^{79}$ René Fédou, Les hommes de loi lyonnais, op. cit. (n. 72), p. 78.

${ }^{80}$ Le 27 août 1500 comparaissent entre autres devant le consulat « monseigneur le courrier » et « monseigneur le visiteur du perier », ce qui semble attester que Louis du Périer n'est alors plus courrier (AML, BB 24, $\mathrm{f}^{\circ} 273 \mathrm{v}^{\circ}$ ).

${ }^{81}$ Caroline Fargeix, Les élites lyonnaises, op. cit. (n. 11), p. 600.

${ }^{82}$ La famille de Varey représente 40 mandats consulaires entre 1417 et 1519, le maximum pour cette période. Suivent ensuite les familles Buatier et de Nyèvre mais qui ne représentent chacune que 23 mandats (Caroline Fargeix, Les élites lyonnaises, op. cit. (n. 11), p. 188).

${ }^{83}$ ADR, 15G358.

${ }^{84}$ «Nobili viro Ludovico de pererio visitatori sallie provencie burgensi lugduni eius compatri carissimo ».

85 «Nobili viro ludovico fantini scuttifero de sancto simphoriano auzonis ».
} 
L'appellation n'indique pas forcément l'état de noblesse ${ }^{86}$ mais au moins un rang social élevé. On ne connaît pas les liens qui unissaient Humbert de Varey et Louis du Périer mais ils semblent particulièrement forts. Varey est certainement l'aîné de du Périer, probablement d'une dizaine d'années et le considère apparemment comme un proche, autant amicalement que socialement.

La vie lyonnaise de Louis du Périer continue après son départ du consulat à l'été 1493. Il participe régulièrement aux assemblées des notables et préside même une réunion du consulat le 13 octobre 1493 où sont présents des conseillers « vieux et nouveaux ». Outre sa présence à des assemblées, il fait quelques apparitions plus marquantes dans les registres consulaires. Le 15 janvier $1494^{87}$, alors que le consulat désigne douze citoyens, six du côté du royaume et six pour l'empire, afin de préparer l'entrée de la reine et " pour assister [les consuls] et leur donner aide, conseil et confort pour la matière de pouvoir trouver et avoir promptement argent pour fournir et faire les frais nécessaires à ladite entrée », « Monseigneur le visiteur Louis du Périer » est le premier nommé pour le côté de l'empire ${ }^{88}$. Le 18 octobre $1495^{89}$ il se présente devant le consulat avec Jean le Maistre et Antoine Buatier en leur nom et en celui d'autres citoyens lyonnais qui semblent avoir signé une sorte de pétition. Louis du Périer est leur porte-parole et réclame le remboursement de deux milles livres tournois versées pour un emprunt du roi. Le terme du prêt est alors échu et les débiteurs ont réclamé à plusieurs reprises leur argent au trésorier de la ville, Jacques de Baillieux, qui les a éconduits. Par la voix de du Périer, ils demandent donc au consulat de contraindre le trésorier à effectuer leur remboursement et menacent d'y « remédier par justice ». La requête s'envenime ensuite du fait d'un différend entre le trésorier et les Le Maistre ${ }^{90}$. Le 20 octobre, les conseillers répondent à la demande de du Périer en convoquant le trésorier avec les documents concernant cet emprunt à une date ultérieure pour laquelle nous n'avons pas de compte-rendu. Le 30 janvier $1496^{91}$, Gaspard du Périer, docteur, apparaît pour la première et unique fois dans la liste des participants à une assemblée de notables aux côtés de son père.

En $1496^{92}$, Louis du Périer a la charge de la bannière de la ville « servans au fait de la guerre et eminent peril» pour le côté de l'empire «tant en son nom que comme tuteur et gouverneur d'Antoine de Varey » en remplacement de Humbert de Varey alors décédé. Le 4 décembre $1496^{93}$, ce tutorat d'Antoine de Varey permet à Louis du Périer de prêter 1200

\footnotetext{
${ }^{86}$ Néanmoins ici Humbert et son fils Antoine sont nobles tout comme Éléonore et normalement l'écuyer puisqu'il s'agit théoriquement d'un titre de noblesse.

${ }^{87}$ AML, BB21, f ${ }^{\circ} 27 \mathrm{r}^{\circ}$.

${ }^{88}$ Jean Rochefort, Humbert Mathieu, Jacques Buyer, Jean de Bourges et Jacques Barondeau suivent. Pour le royaume, sont nommés messire Claude le Charron, docteur procureur du roi, monseigneur le visiteur palmier, Guillaume Baronnat, Jean Rosselet, Étienne Garnier et Étienne Laurencin.

${ }^{89}$ AML, BB22, fo 80.

${ }^{90}$ Jean Le Maistre en profite pour accuser Jacques de Bailleux, en plus de refuser le payement de la dette, d'avoir insulté son fils Pierre Le Maistre, également consul, et tenté de le frapper au cours d'une séance du consulat. Jean le Maistre demande donc le renvoi du trésorier. Les torts devaient être quelque peu partagés puisque les consuls demandent aux deux parties de faire amende honorable et de se raccommoder (AML, BB22, $\mathrm{f}^{\circ} 81 \mathrm{r}^{\circ}$ ).

${ }^{91}$ AML, BB22, $\mathrm{f}^{\circ} 97 \mathrm{r}^{\circ}$.

${ }^{92}$ AML, BB24, $\mathrm{f}^{\circ} 4 \mathrm{v}^{\circ}$. La délibération est insérée avec d'autres lettres et ordonnances dans l'introduction, datée du 26 juin 1496, d'un nouveau volume d'actes municipales sans que la date même de cette décision ne soit vraiment claire.

${ }^{93}$ AML, BB24, $\mathrm{f}^{\circ} 64 \mathrm{v}^{\circ}-65 \mathrm{r}^{\circ}$ : « Pour ce que ladite ville doit et tient a intérets et perte de finance grans sommes de deniers / par faulte de deniers communs lesquels interets fault paier aux marchans estrangiers qui ont prestees lesdites sommes ou autrement iceulx marchans molesteroient ladite ville pour avoir paiement dicelles sommes qui est quant à present impossible a pouvoir fournir a cause des grans et urgens affaires que ladite ville a a
} 
livres tournois à la ville sur les deniers de son pupille. La ville n'a alors plus d'argent mais doit rembourser urgemment des marchands étrangers d'un précédent prêt. La ville s'engage alors à rembourser du Périer ou Antoine de Varey d'ici un an et les douze conseillers se portent garants de l'emprunt sur leurs propres deniers. Le contrat du prêt est signé le 8 décembre suivant et reçu par Georges de La Noyerie, notaire et secrétaire du consulat. Cet emprunt ne connaîtra son épilogue que plus de dix ans plus tard après des péripéties que nous verrons plus loin. Peu après, le 18 décembre $1496^{94}$, Louis du Périer se fait élire pour un troisième mandat consulaire avec Claude Le Charron, Barthélémy Bellièvre, Amé Bullioud, Jacques Buyer et Claude Rochefort pour les années 1497-1498. Curieusement, l'une des premières actions ${ }^{95}$ qu'il entreprend ensuite est de réclamer un remboursement pour son voyage à Montpellier en... 1484 ! Même si le consulat est constamment endetté envers les principaux notables lyonnais, une telle demande plus de douze ans plus tard parait étonnante.

\section{DERNIERES ANNEES : HONNEURS CONSULAIRES ET NOBLESSE}

Louis du Périer prête serment comme conseiller le 7 mai $1497^{96}$. Son taux de présence au consulat, $73 \%$, est à nouveau plutôt élevé en 1497. On peut néanmoins observer une absence totale entre les séances du 17 août et du 29 octobre 1497. Un voyage de deux mois hors de Lyon pour des affaires privées peut éventuellement l'expliquer. L'année 1498 est celle où Louis du Périer a été le plus présent au consulat : son nom est inscrit dans 115 séances, soit un taux de présence de $95 \%$. En 1499, Louis du Périer est à nouveau présent à quasiment toutes les séances jusqu'au 19 mars puis de façon plus irrégulière jusqu'au 14 mai. $\mathrm{Au}$ cours de ces deux années, du Périer laisse à Claude Le Charron, docteur en droit et procureur du roi, la première place au consulat et apparaît à la seconde et donc préside en l'absence de son confrère. Celui-ci est, en particulier, absent d'avril à août 1498. Du Périer n'intervient que dans une affaire spécifique, le 5 septembre $1498^{97}$, alors que les conseillers décident de réviser leurs propres taux d'imposition dont ils se plaignent. À cette occasion, « monseigneur le visiteur ledit du perier pour ce qu'il se dit estre noble et avoir ses lettres de noblesse et quil contribue a ceste cause au daulphine ", se fait exonérer d'impôt pour son meuble mais continue à payer pour ses immeubles sauf pour une maison près des cordeliers pour laquelle existe un contentieux et dont il sera contribuable quand il en jouira. C'est la première fois que Louis du Périer se prévaut de la noblesse et demande à en avoir les privilèges. Il est difficile de savoir d'où il la tient. Le roi avait octroyé la noblesse aux consuls

supporter / mesmement a cause de lemprunct accorde au roy A este advise prendre par emprunct de honnorable homme Loys du perier comme tuteur et des deniers de noble anthoine de varey qui a ce sest accorde pour faire plaisir et servir a ladite ville la somme de douze cens livres tournois payable audit du perier tuteur de varey ou a icellui anthoine de varey dedans ung [an] prouchain venu / Dont les douze conseillers dicelle ville chacun pour sa rate a leurs propres et prives noms se obligeront audit du perier ».

94 AML, BB24, f ${ }^{\circ} 67 \mathrm{r}^{\circ}$.

${ }^{95}$ Le 13 janvier $1497\left(\mathrm{AML}, \mathrm{BB} 24, \mathrm{f}^{\circ} 70 \mathrm{v}^{\circ}\right)$ : «A este passe mandement et descharge au tresorier sur les deniers communs de ladite ville de la somme de vingt cinq livres tournois payable a honnorable homme loys du perier citoyen de lyon pour le remboursement de pareille somme quil a payee a cause dune condempnation faicte par les gens des troys estats de languedoc au moys de may mil iiii $^{c}$ iii $^{\mathrm{xx}}$ et quatre pource que ledit du perier lors envoye de par ladite ville a montpellier appella devant auctentique personne du destriement des foyres comme plus a plain est contenu en la requeste et quictance faictes de ladite matiere quil a exhibees et baillees ». Ce remboursement était pourtant compris dans l'ordonnance de payement adressé au trésorier le 23 décembre 1484 (AML, CC514, pièce 16) pour l'ensemble des frais du voyage à Montpellier. Du Périer s'est-il donc fait rembourser deux fois son amende?

96 AML, BB 24, $\mathrm{f}^{\circ}$ 90r ${ }^{\circ}$.

${ }^{97}$ AML, BB 24, f ${ }^{\circ} 182$. 
de la ville en 1495 mais Louis du Périer n'a pas achevé de mandat depuis cette date et surtout Caroline Fargeix montrait que cet octroi n'avait pas eu d'effet jusqu'au milieu du XVI siècle $^{98}$. Il est de plus le seul consul à présenter cet argument lors de cette séance, dix autres obtenant simplement une réduction de la valeur de leur meuble sans argumenter. Ce serait donc indépendamment de ses fonctions municipales que Louis du Périer obtient des lettres de noblesse apparemment enregistrées au parlement de Dauphiné. La même année ${ }^{99}$, il obtient pour son fils Gaspard le privilège de faire l'oraison doctorale de la Saint-Thomas ${ }^{100}$ lors de la proclamation des nouveaux élus, honneur traditionnellement réservé à un juriste.

En 1500, Louis du Périer est sollicité à plusieurs reprises par le consulat. En avril, il est appelé et donne son avis sur la question de la présidence du consulat alors que deux docteurs en droit ont été élus cette année-là. Le 5 novembre, il revient présider une séance du consulat qui décide, entre autres, que Louis du Périer, Étienne Grollier, Jean Dodieu et Pierre de Bourg porteront le dais lors de l'entrée du cardinal ${ }^{101}$. À la fin de l'année $1500^{102}$, du Périer est élu pour un quatrième mandat au consulat pour 1501-1502. Il fait son serment le 14 février $1501^{103}$ et est ensuite extrêmement présent avec, à nouveau, un taux de présence de $95 \%$ en 1501. De plus, sauf aux rares séances qu'il manque, il préside systématiquement le consulat, aucun gradué en loi n'étant consul à ce moment. La fin de l'année 1500 et le début de 1501 sont marqués par des problèmes d'approvisionnement en blé qui ont fait grimper les prix en ville et par conséquent le mécontentement. Le 15 mars $1501^{104}$, des consuls et des notables avancent de l'argent à la ville pour faire acheter et ramener du blé à Lyon. Louis du Périer contribue à hauteur de 300 livres tournois ${ }^{105}$. Le 21 juillet, Pierre Rennart promet de fournir à la ville 3000 ânées de blé contre une avance immédiate de 1000 francs. Les consuls acceptent et Louis du Périer comme huit autres consuls et notables avancent chacun 100 livres tournois $^{106}$. D'après Caroline Fargeix ${ }^{107}$, Louis du Périer est chargé de l'oraison doctorale de la Saint-Thomas en décembre 1501, trois ans après avoir obtenu cet honneur pour son fils. En 1502, son taux de présence aux séances du consulat est de 93,5\%. Un docteur en loi, Claude Le Charron, a été élu pour 1502-1503 mais il est très rarement présent et Louis du Périer continue de présider habituellement le consulat. Le 12 avril $1502^{108}$, du Périer est envoyé au

\footnotetext{
${ }^{98}$ Caroline Fargeix, Les élites lyonnaises, op. cit. (n. 11), p. 351. Elle montre que, si certains consuls demandent à jouir de ces privilèges, comme Jacques Buyer en 1497 qui fait d'ailleurs partie de ceux ayant obtenu une réduction à cette séance du consulat, aucun anoblissement n'est constaté jusqu'à la fin de la période qu'elle étudie (1520). Le parlement de Paris n'enregistre les lettres royales accordant la noblesse aux consuls de Lyon qu'en 1544.

${ }^{99}$ AML, BB24, $\mathrm{f}^{\circ} 189 \mathrm{r}^{\circ}$.

${ }^{100}$ Sur cette oraison, voir : Caroline Fargeix, Les élites lyonnaises, op. cit. (n. 11), p. 328-333.

101 AML, BB 24, fo $284 \mathrm{v}^{\circ}$.

${ }^{102}$ La proclamation des élections n'a pas été retranscrite dans les délibérations municipales cette année-là.

${ }^{103}$ La séance de son serment est insérée dans les registres municipaux à la date du 14 février 1502 (1501 n.s.) $\left(\mathrm{AML}, \mathrm{BB} 24, \mathrm{f}^{\circ} 348 \mathrm{r}^{\circ}\right)$. Mais le jour de la semaine - dimanche - correspond à 1501 . La crise du blé évoquée ce jour-là renvoie également plus à 1501 de même que les conseillers présents. Comme Louis du Périer est ensuite presque systématiquement présent à partir du 15 février, on peut supposer une erreur d'année lors de la mise au propre. À d'autres endroits des registres municipaux, se trouvent également des problèmes de cohérence chronologique semblables.

${ }^{104}$ AML, BB 24, f $306 v^{\circ}$.

${ }^{105}$ Les autres consuls et notables présents ne contribuent pas tous. Ceux qui le font prêtent entre 100 écus (soit 181 livres et 5 sols tournois) et 500 livres tournois.

${ }^{106} \mathrm{AML}, \mathrm{BB} 24, \mathrm{f}^{\circ} 325 \mathrm{r}^{\circ}$. Le consulat s'engage également à ne pas acheter de blé ailleurs jusqu'à fin août.

${ }^{107}$ Caroline Fargeix, Les élites lyonnaises, op. cit. (n. 11), p. 328. La référence donnée est malheureusement erronée et nous n'avons pas réussi à la retrouver.

108 AML, BB24, f $354 v^{\circ}-355 r^{\circ}$.
} 
conseil de l'archevêque pour évoquer le transport des "pauvres filles repenties » au grand hôpital, proposé par l'évêque auxiliaire de Lyon et accepté par le consulat. En 1503, Louis du Périer quitte rapidement le consulat. On l'y trouve jusqu'en mars mais avec un taux de présence de seulement $60 \%$ jusque-là. Il assiste ensuite à quelques assemblées de notables jusqu'au 5 septembre $1503^{109}$. Cette séance du consulat, où notables et officiers royaux étaient consultés sur la coadministration de l'hôpital de la Chana avec le chapitre Saint-Paul, est la dernière trace de l'existence de Louis du Périer.

Louis du Périer est néanmoins encore évoqué dans l'affaire qui fait apparaître Antoine de Varey dans la vie politique lyonnaise : le remboursement du prêt de 1200 livres tournois consenti par son tuteur en 1496. Antoine de Varey vient réclamer son argent au consulat le 26 mars 1504. La ville n'a alors pas l'argent et temporise, Antoine de Varey s'impatiente et le consulat se souvient subitement que les Varey ont un retard d'impôt de 1600 livres tournois depuis une quinzaine d'années, ce qu'Antoine de Varey conteste en prétextant sa noblesse et celle de son père. Après de longues procédures pendant lesquelles la ville ne verse que 400 livres tournois à Antoine de Varey, les deux parties finissent par s'entendre et se quitter mutuellement par contrat du 4 novembre $1507^{110}$. Au cours des développements de cette longue affaire, les origines du différent sont rappelées à deux reprises : le 27 mars 1506 et le 28 août 1507. Dans cette première occurrence, Louis du Périer n'est pas donné comme mort alors que dans la seconde, il apparaît comme «feu honnorable homme loys du perier ». On peut en conclure que Louis du Périer se retire de la vie publique lyonnaise à la fin de l'année 1503 et meurt probablement à la fin de l'année 1506 ou début 1507 . On peut également ajouter que le 15 avril $1507^{111}$, Antoine de Varey prend la bannière de la ville pour l'empire mais Louis du Périer qui l'avait auparavant depuis 1496 n'est pas mentionné.

Il n'est pas improbable que la « disparition » de Louis du Périer après 1503 soit liée à l'apparition d'Antoine de Varey avec cette affaire du prêt qui lui est dû. Louis du Périer avait réussi une belle opération en mettant à disposition de la ville cette importante somme d'argent qui ne lui appartenait pas, s'attirant certainement les bonnes grâces des consuls de l'époque sans aucun risque. Mais Louis du Périer sait aussi que les caisses de la ville sont constamment vides, qu'il est très impossible de rembourser une telle somme rapidement et surtout que ce sont les consuls qui avancent, souvent sans retour, de l'argent à la ville. Du Périer n'avait aucun intérêt à demander un remboursement qui serait retombé sur lui-même ou sur ses confrères. On ne sait pas exactement quand Antoine de Varey devient majeur mais du Périer a probablement gardé une influence sur la gestion des biens de son ancien pupille et a pu représenter une figure paternelle pour celui-ci. Par ce biais, du Périer a certainement contribué à faire patienter Varey. Si ce n'est pas une simple coïncidence chronologique, la disparition de Louis du Périer a permis la prise d'indépendance d'Antoine de Varey qui devait avoir bien moins de scrupule à réclamer son argent à des notables dont il est bien moins proche que son tuteur. Incapable de payer, la ville a effectivement dû faire appel aux consuls qui s'étaient portés garants du prêt en 1496 et à leurs descendants, a fait tarder l'affaire et trouvé une parade évitant le remboursement de l'intégralité de la somme.

\section{BIENS ET BIBLIOTHEQUE}

\footnotetext{
109 AML, BB24, $\mathrm{f}^{\circ} 421 \mathrm{v}^{\circ}$.

${ }_{110}$ AML, BB 25, f $193-197$. Cette longue affaire est développée au cours des séances des 18 février 1505,15 janvier 1506, 27,28 et 29 mars 1506, 17 et 22 juin 1507, 28 août 1507 et 4 novembre 1507 .

${ }^{111}$ AML, BB25, f $131 v^{\circ}$.
} 
On peut ajouter à ces éléments biographiques les deux relevés fiscaux des Nommées de de 1493 et 1516 qui recensent les biens des habitants de la ville. En $1493^{112}$, Louis du Périer possède une maison haute, moyenne et basse avec son entrée rue Mercière et des boutiques dans la rue allant de Saint-Nizier au pont de Saône d'une valeur de 1320 livres tournois, une grange avec deux corps de bâtiment et un jardin près de la porte des Cordeliers d'une valeur de 80 livres tournois et un meuble estimé à 400 livres tournois, soit, au total, une assise fiscale de 1800 livres tournois ${ }^{113}$. Les boutiques et une part de la maison de la rue Mercière étaient louées pour 300 livres tournois par an à Janon Colombier, épicier. En 1528-1529114, on retrouve une rubrique consacrée aux « hoirs feu Loys du perier» dans ces documents fiscaux. Ils possèdent toujours la maison de la rue Mercière qui est décrite comme une maison haute, moyenne et basse avec deux corps de bâtiment, l'un sur la rue allant du pont à Saint-Nizier, l'autre occupant l'espace entre la rue Mercière et la Saône. Ce dernier appartient à François du Périer et Marguerite Baronnat, veuve de Louis du Périer alors que le premier corps appartient à Gaspard du Périer. Gaspard du Périer a deux locataires, Marguerite et François en ont trois. François du Périer a acquis en outre une autre maison contigüe à la première. Il n'y a en revanche aucune estimation financière. La nommée de 1493 fait rentrer Louis du Périer dans le groupe des Lyonnais les plus riches ${ }^{115}$ sans qu'il ne le soit excessivement (Guillaume Baronnat est estimé à 2603 livres, Étienne Laurencin à 4768...). La liste de ses biens (du moins à Lyon et dans le Lyonnais) est étonnamment restreinte alors que nombre de riches Lyonnais multiplient les immeubles de rapport et les pensions assises sur l'immobilier lyonnais. Sa demeure principale est extrêmement bien cotée (la plupart des immeubles lyonnais ne dépassent pas les 100 livres tournois de valeur) mais elle est aussi très bien située, au cœur de Lyon et à quelques pas de Saint-Nizier, centre du pouvoir consulaire, et était certainement grande et bien bâtie. Le loyer annuel - 300 livres tournois - payé pour une partie de cette demeure et ses boutiques confirment d'ailleurs l'attractivité, en particulier commerciale, de l'emplacement. La grange près des Cordeliers est probablement la maison pour laquelle il est en procès en 1498 quand il revendique sa noblesse. Elle est apparemment perdue ou vendue avant 1528 .

Ce portrait de Louis du Périer ne peut être complet sans la mention des six manuscrits qu'il a possédés: des Opera de Virgile ${ }^{116}$, les Dicta et facta memorabilia de Valère Maxime $^{117}$ en latin avec la traduction française de Simon de Hesdin et de Nicolas de Gonesse, La Bouquechardière de Jean de Courcy ${ }^{118}$, l'Ovide moralisé ${ }^{119}$, une Histoire ancienne ${ }^{120}$ et le Roman de Jules César ${ }^{121}$. Ces manuscrits, dont plusieurs ont été richement enluminés par les

\footnotetext{
112 AML, CC 11, $\mathrm{f}^{\circ} 77 \mathrm{r}^{\circ}$.

${ }^{113}$ Les estimations des Nommées sont truquées et ne correspondent pas à une valeur réelle. Les estimations sont faites à partir d'un immeuble de référence très sous-estimé afin de minorer la richesse de la ville aux yeux du roi ; voir : Jean Deniau, Les nommées des habitants de Lyon en 1446, Félix Alcan, Paris, 1930, p. 42-43.

${ }^{114}$ AML, CC 39, fo 10.

115 Les artisans sont généralement estimés à quelques dizaines de livres tournois, les marchands et bourgeois à quelques centaines. Rares sont les Lyonnais à dépasser les 1000 livres tournois d'estime.

116 Paris, BnF, ms lat. 8200. Pour le rattachement à du Périer, voir: François Avril, Nicole Reynaud, Les manuscrits à peintures en France : 1440-1520, Paris, Flammarion, 1995, p. 201.

${ }^{117}$ Chantilly, musée Condé, mss 833-834.

118 Genève, BPU, ms. fr. 70.

${ }^{119}$ Paris, BnF, ms fr. 374.

${ }^{120}$ Bruxelles, Bibliothèque royale de Belgique, ms 10175.

${ }^{121}$ Paris, BnF, ms fr. 1457.
} 
meilleurs ateliers lyonnais de l'époque, illustrent le goût de Louis du Périer pour la littérature et en particulier pour l'histoire ${ }^{122}$.

\section{ANALYSE D'UNE CARRIERE LYONNAISE REUSSIE}

L'ensemble des éléments rassemblés ici permettent de se faire une idée plus précise de la personne de Louis du Périer. Tout d'abord, c'est un Lyonnais, servant sa ville et parfaitement intégré dans la société lyonnaise qui semble le reconnaître comme un de ses membres les plus éminents et lui accorde nombre d'honneurs. Il est toujours donné comme « citoyen de Lyon » et se marie dans la bourgeoisie lyonnaise. Sa naissance dans le Lyonnais ou d'une famille lyonnaise paraît extrêmement probable. Il faut donc réfuter toute la littérature qui le considère au pire comme Provençal ${ }^{123}$, au mieux comme Provençolyonnais ${ }^{124}$. Au vu de ses taux de présence à Lyon, son office provençal apparaît quasiment comme un «emploi fictif ». C'est un citoyen extrêmement aisé, appartenant aux couches sociales supérieures de la population. Il semble être dans cette partie du patriciat lyonnais qui se trouve alors aux frontières de la bourgeoisie et de la noblesse. Il s'investit dans la gestion de la ville, est liée socialement à la bourgeoisie mais occupe des offices «nobles » comme ceux de visiteur du sel ou de courrier et finit par obtenir des lettres de noblesse, apparemment sans lien avec ses fonctions consulaires. Il ne semble pas avoir vraiment de métier : on le voit voyager pour la ville de nombreux mois sans que cela pose problème et parmi les métiers lyonnais, il est élu avec les rentiers. Mais il ne possède ni pension, ni immeuble de rapport à Lyon et dans le Lyonnais ${ }^{125}$. Ses fonds se trouvent par conséquent ailleurs. On peut supposer qu'il possède des terres comme d'autres bourgeois quasi-nobles, peut-être en Dauphiné puisque c'est là qu'il fait enregistrer ses lettres de noblesse. Il a ainsi un mode de vie qui se rapproche aussi de la noblesse. En bien des aspects, il est comparable au bien plus connu Humbert de Varey qui a également servi le consulat, fréquenté la bourgeoisie lyonnaise ${ }^{126}$, été courrier de Lyon, possédé des offices royaux, été anobli et qui possède une seigneurie et des terres. Celui-ci le traite d'ailleurs en égal dans son testament. Humbert de Varey et Louis du Périer représentent certainement cette partie du patriciat lyonnais, pas forcément la plus riche mais qui a réussi à transformer ses ressources sociales et matérielles pour se projeter d'une activité urbaine bourgeoise aux bases d'une vie noble dans laquelle leurs enfants grandiront. Avant l'automatisation de l'anoblissement des consuls qui va profondément bouleverser l'équilibre de la bourgeoisie lyonnaise au $\mathrm{XVI}^{\mathrm{e}}$ siècle, ce sont des parcours assez rares qui concernent des individus bien implantés dans la ville.

Cette insertion dans la bourgeoisie lyonnaise se lit d'ailleurs dans sa brillante carrière consulaire. Avec quatre mandats, il fait partie de l'élite consulaire mais sans compter parmi les consuls les plus élus. En effet $7 \%$ des consuls font quatre mandats entre 1417 et 1519

\footnotetext{
122 Nous reviendrons sur ces manuscrits étudiés au cours de notre thèse dans futur article centré sur la bibliothèque de du Périer.

123 « Amateur de Provence» (Hippolyte Aubert, Notices sur les manuscrits Petau conservés à la bibliothèque de Genève, Bibliothèque de l'école des chartes, 1909, tome 70, p. 485-486), «Loys du Périer d'Aix » (Marguerite Debae, La bibliothèque de Marguerite d'Autriche, Louvain ; Paris, Peeters, 1995).

124 «bibliophile lyonnais-provençal » (François Avril, Nicole Reynaud, Les manuscrits à peintures, op. cit. (n. 116), p. 358).

${ }^{125}$ Les nommées prennent en compte les propriétés du Lyonnais. On conserve également quelques registres des propriétés des habitants de Lyon dans le Lyonnais (AML, CC15-19, 49-56) mais Louis du Périer n'y figure pas.

${ }^{126}$ Quand Antoine de Varey vient contester les impôts que la ville lui réclame pour lui et son père, les consuls lui rappelle que son père est un des leurs, qu'il a fait des affaires, marchandé en ville et est avant tout un bourgeois. Au contraire Antoine de Varey, seigneur de Balmont, est né noble de père noble et a toujours vécu noblement.
} 
quand $19,5 \%$ en font plus de quatre (alors que $44 \%$ n'en font qu'un seul) ${ }^{127}$. Au-delà de la quantité de mandats, l'importance de du Périer peut se mesurer aussi qualitativement. Dans son analyse du consulat lyonnais, Caroline Fargeix distinguait des élus pour qui la charge consulaire est avant tout une récompense de ceux qui, issus du patriciat lyonnais, exerçaient réellement le pouvoir ${ }^{128}$. Louis du Périer fait clairement partie du second groupe et en est même probablement l'un des membres les plus éminents. Son parcours semble se faire en deux temps. Dans les années 1480, il effectue son premier mandat où son rang dans l'ordre des consuls est assez médiocre ${ }^{129}$ mais le vote du 30 juillet 1487 montre qu'il fait partie de ceux qui exercent réellement le pouvoir. À côté, il effectue pour la ville de nombreuses missions dont certaines paraissent être d'importance, ce qui montre la confiance qu'on lui accorde dès cette époque. C'est l'occasion pour lui de montrer sa compétence et sa volonté de servir la ville. Par la suite, à partir des années 1490, il cesse ses voyages mais apparaît en première ou seconde position dans la liste des consuls, précédé uniquement par des docteurs en droit. À ce titre, il exerce fréquemment la présidence du consulat. Cette présidence ainsi que l'oraison doctorale de la Saint-Thomas qu'il réalise en 1501 font partie des principaux honneurs de la ville, théoriquement réservés aux gradués en droit mais qu'il arrive néanmoins à obtenir. Ses exceptionnels taux de présence aux séances du consulat confirment son importance dans celui-ci. Caroline Fargeix montre également que l'élection comme maître des métiers permet aux principaux bourgeois d'être consultés dans les assemblées de notables et de rester dans les affaires entre deux mandats consulaires. Louis du Périer n'exerce qu'une seule maîtrise de métier, à la suite de son premier mandat, ce qui ne l'empêche pas d'être présent à la très grande majorité des assemblées de notable par la suite. Il paraît donc avoir été un élément pratiquement incontournable de la vie publique lyonnaise dès son premier mandat, ce qui peut aussi expliquer qu'il n'ait pas brigué plus de mandats de consul ou de maître de métiers. Il en connaît d'ailleurs les inconvénients et n'est pas le dernier à réclamer des remboursements. Il ne fait pas partie des consuls les plus généreux et le prêt le plus important qu'il concède à la ville n'est pas de ses propres deniers mais de ceux de son pupille. Les seules contributions d'importance qu'on lui connaît sont faites en 1501 mais, à ce moment, il préside le consulat et peut difficilement demander aux consuls et notables d'avancer de l'argent pour acheter du blé sans donner l'exemple. À bien des égards, Louis du Périer est certainement l'un des hommes les plus influents de la bourgeoisie lyonnaise du dernier quart $\mathrm{du} \mathrm{XV}^{\mathrm{e}}$ siècle avec l'une des plus brillantes carrières au sein du consulat.

La période de sa vie entre son mariage en 1465 et sa carrière consulaire dans les années 1480 reste mystérieuse. Il paraît occuper alors des offices royaux. Cela démontre des relations sociales avec la cour qui peuvent être également l'une des raisons du choix de Louis du Périer pour des missions diplomatiques en faveur de la ville. Au cours de l'une d'elle, il obtient du roi la charge de visiteur des gabelles à sel de Provence qu'il prend réellement en 1489. Cet office, d'une grande importance et considéré comme noble, est à la tête de l'administration du sel dans le comté de Provence avec des pouvoirs de justice dans la répression des fraudes. On peut néanmoins s'interroger sur l'exercice réel de la charge par Louis du Périer. De 1489 à sa mort, soit une quinzaine d'années, il effectue trois mandats

\footnotetext{
${ }^{127}$ Caroline Fargeix, Les élites lyonnaises, op. cit. (n. 11), p. 187. La répartition exacte par nombre de mandat se trouve dans sa thèse (disponible en ligne).

${ }^{128}$ Caroline Fargeix, Les élites lyonnaises, op. cit. (n. 11), p. 187.

${ }^{129}$ Lors de ces quelques jours de présence en 1486, parmi les présents, seuls François de Genas et François de Rivoire sont nommés après lui.
} 
consulaires représentant six années pendant lesquelles, hors une absence d'août à octobre 1497, on peine à trouver dix jours consécutifs pendant lesquels sa présence n'est pas attestée à Lyon. Sa présence dans la ville est plus difficile à mesurer quand il n'est pas élu mais sa présence régulière aux assemblées de notables atteste qu'il y réside habituellement. Déjà dans les années 1470, quand il était receveur en Albigeois, la confection pour lui d'un manuscrit écrit par un scribe lyonnais et enluminé par un atelier lyonnais pouvait faire douter d'une réelle résidence dans la province de son office. Son titre d'officier royal lui apporte en tout cas une grande considération à Lyon et contribue peut-être à sa présence lors des assemblées de notables où l'on trouve plusieurs officiers royaux même si, à part lui, tous ont un office pour le Lyonnais ${ }^{130}$. Son influence à la cour est aussi discernable dans la nomination de son fils Gaspard comme conseiller au parlement d'Aix quelques années après avoir tenté de l'installer à la cour séculière de Lyon. Gaspard du Périer fait en effet partie des onze premiers conseillers nommés à la création du parlement d'Aix par Louis XII par édit royal donné à Lyon le 10 juillet 1501. La présence du roi à Lyon a certainement été une occasion inespérée pour obtenir un office prestigieux à son fils. César de Nostredame, dans son Histoire et chronique de Provence indique que c'est Anne de Bretagne qui fit donner une charge de conseiller à Gaspard lors de l'érection du parlement ${ }^{131}$. Outre sa place importante dans la vie politique lyonnaise, il faut donc créditer Louis du Périer d'une certaine influence à la cour de France dès les années 1470, qui lui permit d'obtenir pour lui-même et pour son fils des offices importants. On peut également suggérer que les lettres de noblesse évoquées en 1498 sont aussi d'origine royale.

\section{L’ASCENDANCE DE LOUIS : UN THOMASSIN DU PERIER ?}

Les patronymes Perier/Perrier et leurs dérivés sont fréquents en France. En langue d'oïl, on les rapproche du terme de carrière ou du métier de carrier, en langue d'oc du poirier $^{132}$. Les homonymes rendent compliqués toute recherche sur les origines de la famille du Périer même s'il faut souligner que la particule «du» est relativement rare et que l'ensemble des documents évoquant Louis du Périer utilise une unique graphie pour ce nom (du perier en français, de pererio en latin). La structure du patronyme évoque plutôt un lien avec toponyme, soit la proximité d'une carrière, soit un lieu-dit «Le Périer » dont la famille pourrait être originaire ou posséder.

Comme nous l'avons signalé en introduction la parentèle de Louis du Périer est inconnue $^{133}$. Pis, le patronyme semble n'être jamais apparu avant lui ni dans les travaux sur Lyon au Moyen Âge ni dans les transcriptions de pièces d'archives existantes. La situation est

\footnotetext{
${ }^{130}$ Etant donné la multiplicité des pouvoirs et des juridictions à Lyon, l'invitation des officiers de l'archevêque et des officiers du roi pour le Lyonnais dans les délibérations se comprend pour une action efficace. La présence du visiteur du sel de Provence pour ce seul titre est moins compréhensible.

${ }^{131}$ César de Nostredame, Histoire et chronique de Provence, Lyon, Simon Rigaud, 1624, p. 689. Néanmoins, le témoignage de César de Nostredame, bien que familier de la famille du Périer au XVII ${ }^{\mathrm{e}}$ siècle, est douteux pour ce qui relève du passé. Ses considérations sur l'origine de la famille du Périer au Moyen Âge (rattachement à la famille bretonne homonyme) sont totalement erronées.

${ }^{132}$ Une famille de Perier a ainsi des armes parlantes avec un poirier en Provence.

${ }^{133}$ On trouve parfois dans certaines généalogies anciennes de la famille Bellièvre une Françoise du Perrier, sœur d'un certain Jean du Perrier, élu consul de Lyon en 1483, comme épouse de Huguenin Bellièvre. Après vérification, il s'agit en fait de Jean du Peyrat dont la famille est bien distincte de celle des du Périer. Les armes de la famille du Peyrat sont : d'azur au château d'or.
} 
assez paradoxale dans la mesure où sa carrière lyonnaise atteste d'une excellente insertion dans la bourgeoisie lyonnaise. Une origine étrangère est assez improbable, de même qu'une ascension sociale fulgurante qui le ferait apparaitre dans les registres lyonnais au contraire de ses ascendants. Les travaux sur le patriciat lyonnais au $X^{\mathrm{e}}$ siècle montrent qu'il s'agit d'un milieu extrêmement fermé qui ne s'ouvre que lentement et sur plusieurs générations à des homines novi ${ }^{134}$. Du Périer n'a d'ailleurs ni grade universitaire, ni fortune considérable, ni stature intellectuelle, ni activité bienfaisante qui pourrait justifier une exception. Sa carrière consulaire n'est d'ailleurs pas vraiment ascendante comme on pourrait s'y attendre de la part d'un homo novus puisque dès la fin de son premier mandat, il apparaît au sommet de la hiérarchie. La famille Bellièvre représente une des ascensions sociales remarquables de cette époque mais on connaît ses origines en Bresse et ni Hugonin, ni même son neveu Barthélémy qui constitue la seconde génération, n'a une stature comparable à celle de Louis du Périer. L'hypothèse qui paraît donc la plus vraisemblable est qu'il pourrait s'agir d'un patronyme adopté par une branche cadette d'une des grandes familles lyonnaises de cette époque. Cette hypothèse permet d'expliquer à la fois l'absence du patronyme avant Louis et la place de celui-ci dans la société lyonnaise. Le patronyme n'a évidemment pas au Moyen Âge la même constance qu'aujourd'hui et il n'est pas rare de trouver dans les archives lyonnaises des patronymes en alias ou des fils n'ayant pas le même patronyme que leur père. La façon de désigner les gens peut aussi changer ; c'est le cas, par exemple, du pupille de Louis du Périer, appelé «de Balmont» dans les registres municipaux d'après le nom de sa seigneurie, alors que son père était appelé « de Varey ». Dans la bourgeoisie aux frontières de la noblesse, classe à laquelle appartient Louis du Périer, il n'est d'ailleurs pas inhabituel de prendre le nom d'une de ses terres par imitation de la noblesse. On peut supposer que c'est ce qui s'est passé dans le cas des du Périer et que Louis est le premier pour lequel ce nouveau nom d'usage se soit imposé. Louis du Périer est en effet un propriétaire terrien et son nom correspond probablement à un toponyme.

À quelle famille peut-on essayer de rattacher Louis du Périer? Les familles consulaires lyonnaises du Moyen Âge sont malheureusement très mal connues et leurs généalogies très incertaines. La vie de Louis du Périer présente manifestement des liens avec la famille de Varey. L'élément le plus marquant est certainement le testament de Humbert de Varey qui désigne Louis du Périer comme son exécuteur testamentaire et comme tuteur de son fils, place généralement dévolue à un oncle. On peut ajouter que c'est Humbert de Varey qui lance la carrière municipale de Louis du Périer en le proposant pour une mission à Montpellier en 1484, qu'ils appartiennent exactement à la même classe sociale mais aussi que la moitié des courriers de Lyon, office occupé par Humbert vers 1489 et par Louis vers 1493, que l'on connaît pour le $\mathrm{XV}^{\mathrm{e}}$ siècle sont des Varey. Toutefois, dans son testament, Humbert de Varey explicite le lien familial avec certains des légataires mais ne dit absolument rien sur Louis du Périer, ce qui paraît exclure un lien familial direct. Un lien familial plus lointain ou par alliance peut être envisagé.

[Image 1. Légende : Armes de Louis du Périer, Genève, Bibliothèque de Genève, ms. fr. 70/1, f. 4 (www.e-codices.ch) ]

Outre le patronyme, parmi les éléments constitutifs d'un lignage au Moyen Âge se trouvent les armes. Même si elles peuvent aussi être changées, elles sont probablement même

${ }^{134}$ Caroline Fargeix, Les élites lyonnaises, op. cit. (n. 11), p. 187-195. 
plus fixes que le patronyme au Moyen Âge. Louis du Périer porte des armes d'azur à la bande d'or, accompagnée en chef d'une tête de lion arrachée du même, lampassée de gueules et couronnée d'argent, et à la bordure engrêlée de gueules ${ }^{135}$. Or, il se trouve que ces armes sont extrêmement proches de celles d'une autre famille lyonnaise : les Thomassin qui portent d'azur à la bande d'or accompagnée en chef d'une tête arrachée du même, rapprochement qui ne semble pas avoir été fait jusque-là. La différence notable entre les deux est la bordure de gueules qui, en héraldique française, fait partie des marques de brisure les plus employées. En effet, théoriquement, seul le chef de famille porte les armes pleines, les autres membres puis, après eux, les branches cadettes, brisant ces armes de manière assez diverses ${ }^{136}$. L'application réelle de ces principes est souvent difficile à prouver mais il s'avère que l'on peut attester de l'utilisation concrète de brisures chez les Thomassin ${ }^{137}$ comme chez les du Périer $^{138}$. La bordure engrêlée de gueules est une variante relativement rare de la bordure de gueules mais il en existe un exemple célèbre, celui du duc Jean de Berry, troisième fils du roi Jean II de France, qui portait de France à la bordure engrêlée de gueules, armes ensuite associées au duché de Berry. D'autres princes de la fin du XIV et du XV ${ }^{\mathrm{e}}$ siècle portent également des bordures engrêlées comme brisure ${ }^{139}$. En héraldique comme en d'autres matières, les exemples princiers ayant tendance à être reproduits par les couches inférieures, il ne paraît pas étonnant que l'on trouve ce type de brisure dans des familles lyonnaises au milieu du $\mathrm{XV}^{\mathrm{e}}$ siècle. Les armes de du Périer apparaissent donc assez clairement comme une brisure de celles de Thomassin et ce d'autant plus que le motif central est rarissime, ce qui écarte aussi la possibilité d'une quasi-homonymie accidentelle. Les armes, comme les patronymes, peuvent en effet présenter des homonymies entre familles n'ayant aucun lien du fait de l'utilisation très fréquente de certains meubles. Ici, la bande comme le lion sont des meubles très usités; la tête de lion arrachée est déjà plus rare mais le motif de la bande accompagnée en chef d'une tête de lion arrachée est absolument exceptionnel. Les armoriaux du Lyonnais, Forez et Beaujolais ne donnent que du Périer et Thomassin avec ce motif. Même si une recherche sur l'ensemble de l'Europe peut difficilement être exhaustive, nous n'avons trouvé dans l'ensemble des ressources disponibles que peu de familles avec ce motif et la plupart ont d'autres émaux ou d'autres meubles qui les rendent bien plus distinctes ${ }^{140}$. On peut

135 D'après les armes peintes sur le manuscrit de la Bouquechardière. Les armoriaux modernes donnent généralement à la famille du Périer de Provence une tête de lion couronnée d'or ou d'argent et lampassée de gueules. Ces détails du lion sont des licences artistiques à la fin du Moyen Âge et leur usage ne se stabilisent qu'au XVII ${ }^{\mathrm{e}}$ siècle (Michel Pastoureau, Traité d'héraldique, 5 édition, Paris, Picard, 2008, p. 142). La tête de lion entière est parfois aussi entièrement d'argent.

${ }^{136}$ Michel Pastoureau, Traité d'héraldique, op. cit. (n. 135), p. 177-187.

${ }^{137}$ Sur le manuscrit français 423 de la bibliothèque nationale de France, on trouve avec une main du XV siècle un ex-libris des Thomassin accompagné de leurs armes brisées par l'ajout d'une étoile sur la bande.

${ }^{138}$ François du Périer, probable fils cadet de Louis, brise ses armes par changement de couleur de la bordure engrêlée qui devient d'or dans les registres des armoiries consulaires de Lyon. Cette existence de brisures chez ces deux familles au XV est particulièrement notable dans la mesure où la pratique de la brisure tend à disparaître en dehors des familles princières à partir du XIV siècle : Michel Pastoureau, Traité d'héraldique, op. cit. (n. 135), p. 180.

${ }^{139}$ Outre Jean du Berry, on peut citer les princes Jean de France (1398-1417), de France à la bordure engrêlée et componée d'argent et de gueules, Charles de France (1146-1472), de France à la bordure engrêlée de gueules, ou au-delà de la famille royale Richard d'Étampes (1395-1438), de Bretagne à la bordure engrêlée de gueules.

${ }^{140}$ Nous avons trouvé les familles de Chalvet (de gueules, à une bande d'or, chargée de trois croisettes du champ, accompagnée en chef d'une tête de lion d'or et en pointe d'une rose du même), Caboud (d'argent à la bande d'azur, chargée de trois étoiles du champ et accompagnée en chef d'une tête de lion de gueules et en pointe d'un croissant du même) et Casimir-Perier (semblables aux armes des Thomassin; l'attribution est probablement erronée, par confusion avec nos du Périer, la famille bourgeoise des Casimir-Perier, notable au XIX ${ }^{\mathrm{e}}$ siècle, n'ayant apparemment jamais porté d'armes). 
exclure également l'usurpation d'armes qui paraît difficile quand la famille visée est une des plus notables de sa propre ville. Tout ceci permet d'affirmer que Louis du Périer porte très certainement des armes de Thomassin à la bande engrêlée de gueules et qu'il fait partie d'une branche cadette de cette famille.

Les Thomassin sont l'une des principales familles lyonnaises du $\mathrm{XV}^{\mathrm{e}}$ siècle. Le premier consul de cette famille est Pierre Thomassin en 1395 et de cette date jusqu'à 1500, chaque génération accède au consulat, faisant au total douze mandats ${ }^{141}$. Ils comptent parmi les plus anciennes familles lyonnaises d'après les mémoires de Claude Bellièvre qui rapporte dans la première moitié du XVI ${ }^{\mathrm{e}}$ siècle les propos de son père ${ }^{142}$. C'est une famille de drapiers et de notaires dont l'un des membres les plus connu au $\mathrm{XV}^{\mathrm{e}}$ siècle est probablement Mathieu Thomassin (vers 1391-après 1463), docteur en droit, conseiller au conseil delphinal, président de la chambre des comptes de Grenoble et auteur du Registre delphinal, écrit vers 1446-1456. Il est ainsi un proche collaborateur du dauphin Louis, futur roi Louis XI. Par lui, la famille Thomassin a des attaches en Dauphiné et surtout une certaine proximité avec le souverain. Est-ce donc une coïncidence si Louis du Périer, après avoir servi le roi, entame une carrière consulaire dans sa ville en 1484, quelques mois après la mort de Louis $\mathrm{XI}^{143}$ ? Ou bien est-ce une reconversion stratégique alors que s'amorce la régence ? Les attaches et éventuelles terres de Louis du Périer en Dauphiné peuvent aussi trouver ainsi une explication. Il peut être tentant de faire de ce Matthieu Thomassin le père de Louis du Périer. Rien ne s'y oppose mais rien n'appuie non plus cette hypothèse. Les explications peuvent tenir également s'il ne s'agit que d'un vague cousin ou d'un neveu. Ces attaches à la cour ont pu être réactivés par la suite dans les années 1480 au cours des voyages de Louis du Périer et lui ont permis d'obtenir des postes prestigieux pour lui et son fils Gaspard. Dans la branche aînée des Thomassin, Claude Thomassin est à peu près contemporain de Louis du Périer et obtient lui aussi d'importants offices royaux : il est élu du Lyonnais en 1479-1480, lieutenant du capitaine de la ville en 1491 , lieutenant du procureur du roi en 1496, conseiller du roi et conservateur des foires ${ }^{144}$ en 1502 et enfin capitaine de la ville en 1510. Il possède des appuis à la cour : le célèbre écrivain Jean Lemaire de Belges témoigne en 1509 que c'est grâce à Claude Thomassin qu'il obtient des privilèges de la Chancellerie de France ${ }^{145}$ et en 1511 , la ville lui demande d'aller à Paris parce qu'il a «très bon introite chez messires les chancellier ${ }^{146}$ et trésorier général ${ }^{147}$. Claude Thomassin fait cinq mandats consulaires en 1484-1485, 1488-1489, 1504-1505, 1511 1512 et 1516, année pendant laquelle il meurt sans achever son mandat. Il est également compté dans les terriers ${ }^{148}$ parmi les métiers lyonnais et on peut attester qu'il possède des

\footnotetext{
${ }^{141}$ Après Pierre, on a un autre Pierre Thomassin, puis Claude son fils, contemporain de Louis du Périer, puis, au début du XVI ${ }^{\mathrm{e}}$ siècle Bonaventure Thomassin, fils de Claude.

${ }^{142}$ Claude Bellièvre, Charles Perrat (éd.), Souvenirs de voyages en Italie et en Orient, Genève, E. Droz, 1956, p. 69.

${ }^{143}$ Louis XI meurt le 30 août 1483.

144 «Le titre exact du conservateur est, au XVI ${ }^{\mathrm{e}}$ siècle, «Juge, gardien, et conservateur des privilèges royaux des foires de Lyon ». La fonction est des plus élevées ; son titulaire est l'un des personnages les plus importants de la ville » : Marc Brésard, Les foires de Lyon aux XVe et XVI siècles, Paris, Auguste Picard, 1914, p. 299.

145 Jean Lemaire de Belges, Anne Schoysman (éd.), La légende des Vénitiens (1509), Bruxelles, Académie royale de Belgique, 1999, p. LXX.

${ }^{146}$ En 1511, comme en 1509, le chancelier de France est Jean de Ganay.

${ }^{147}$ AML, BB 28, f 262.

${ }^{148}$ Il en est le représentant en 1490, 1496, 1506, 1513.
} 
terres, en particulier à Corbas et à Chandieu dans le Dauphiné proche de Lyon ${ }^{149}$. Les Thomassin, comme les Varey ou Louis du Périer, sont à ce moment aux frontières de la bourgeoisie et de la noblesse. Claude Thomassin est au début du XVI ${ }^{\mathrm{e}}$ siècle chevalier et seigneur de Dommartin mais ce n'est qu'en 1515 qu'il se présente devant le consulat pour se voir exempté d'impôt comme noble ${ }^{150}$. Son fils, Bonaventure, à l'instar de Gaspard du Périer, devient parlementaire, obtenant un office au parlement de Paris puis la présidence du parlement de Grenoble. On peut donc trouver des parallèles assez forts entre les carrières de Louis du Périer et de Claude Thomassin ce qui renforce une proximité qui n'est jamais explicite.

De plus, on peut noter que Claude Thomassin et Louis du Périer ne sont jamais élus ensemble alors qu'ils sont actifs au même moment. Ils alternent même leur présence au consulat entre 1484 et $1489^{151}$. Or les notables lyonnais évitent généralement d'élire ensemble des membres d'une même famille. C'est même théoriquement interdit d'après les statuts du consulat que l'on connaît ${ }^{152}$. Par ailleurs, le fils de Claude, Bonaventure Thomassin, est appelé dans certains documents par un autre nom que celui de son père. On le retrouve en effet sous celui de Bonaventure de Saint-Barthélémy, du nom de sa mère, issue d'une famille bourgeoise anoblie.

Les Thomassin sont de même assez intéressés par les livres. On en connaît quelquesuns comme un manuscrit des œuvres d'Alain Chartier ${ }^{153}$ aux armes de la famille Thomassin, daté du milieu du $\mathrm{XV}^{\mathrm{e}}$ siècle et enluminé par le maître du roman de la rose ${ }^{154}$ qui a également travaillé pour Louis du Périer, mais aussi un manuscrit de vies de saints écrit au XIV siècle avec une table du $\mathrm{XV}^{\mathrm{e}}$ siècle sur laquelle se trouve un ex-libris et les armes des Thomassin (Paris, BnF, fr. 423) $)^{155}$, ainsi qu'un incunable, une Biblia imprimée par Leonhard Wild à Venise en 1478 et ornée aux armes de la famille (Moulins, BM, Inc $\left.4^{\circ} 28338\right)^{156}$. On ne peut restituer des livres qu'à peu de familles lyonnaises de cette époque. Aucun, par exemple, n'est connu pour la famille de Varey. Claude Thomassin est en outre un des protecteurs de Jean Lemaire de Belges. Ce dernier lui adresse une Péroration de l'acteur imprimée avec l'édition princeps de la Légende des Vénitiens par Jean de Vingle en 1509. Un certain goût pour les Lettres et les livres existe chez les Thomassin, ce qui peut être mis en relation avec la bibliophilie connue de Louis du Périer et ses descendants.

L'insertion de Louis du Périer dans la famille Thomassin paraît donc relativement cohérente par rapport à leur milieu social, leurs relations ou leurs attaches diverses.

\footnotetext{
149 Actuelles communes de Corbas (Rhône) et de Saint-Pierre-de-Chandieu (Rhône), sur la rive gauche du Rhône.

${ }^{150}$ AML, BB $34 \mathrm{f}^{\circ} 43$.

151 Claude est consul en 1484-85, Louis en 1486-87 puis Claude à nouveau en 1488-89. Louis l'est ensuite l'année suivante en 1491-92.

${ }^{152}$ Caroline Fargeix, Les élites lyonnaises, op. cit. (n. 11), p. 180.1

${ }^{153}$ Berlin, Kupferstichkabinett, ms. 78 C 7.

${ }^{154}$ François Avril, Nicole Reynaud, Les manuscrits à peintures en France : 1440-1520, Paris, Flammarion, 1995, pp. 199-200.

${ }^{155}$ Sur le recto du premier folio se trouve l'inscription «nissamohT » (Thomassin à l'envers) et les armes de Thomassin brisées d'une étoile ajoutée sur la bande.

${ }^{156}$ Le Catalogue régional des incunables des bibliothèques publiques de France (tome XVI) indique qu'il s'agit de la branche bourbonnaise des Thomassin mais celle-ci n'apparaît qu'au cours du XVI siècle alors que le décor peint est du XV $\mathrm{V}^{\mathrm{e}}$ siècle, c'est-à-dire au moment où les Thomassin sont encore à Lyon.
} 
Les documents d'archives mentionnent clairement Gaspard du Périer comme fils de Louis. En 1493, quand son père le propose comme juge de la cour séculière, il a 25 ans, ce qui le ferait naître en 1468, trois ans après le mariage de Louis et d'Anne Tèze qui est désignée comme sa mère. Il est docteur dans les deux droits de l'université de Pise et obtient en 1501 un des onze offices de conseillers du parlement d'Aix nouvellement créé. Il se marie, probablement avant de partir à Aix, avec Sybille Dupré, aussi issue d'une famille consulaire lyonnaise, et meurt en 1530. C'est lui qui installe véritablement la famille du Périer en Provence où il s'installe pour l'exercice de son office de parlementaire. Vers 1510, il fait bâtir l'hôtel du Périer, qui existe encore aujourd'hui et est resté dans la famille du Périer jusqu'à la fin $\mathrm{du} \mathrm{XVIII}^{\mathrm{e}}$ siècle, sur la place des Prêcheurs à Aix-en-Provence. Les registres fiscaux lyonnais montrent qu'il continue néanmoins à posséder une partie de la maison familiale à Lyon jusqu'à sa mort ${ }^{157}$. La descendance de Gaspard du Périer est bien connue et documentée à l'époque moderne et nous ne reviendrons pas dessus. Elle fait partie de la noblesse provençale et prend au XVIII ${ }^{\mathrm{e}}$ siècle le titre de marquis du Périer, avant de s'éteindre en 1801 pour la ligne directe. Parmi les personnages notables de la descendance de Gaspard du Périer, on peut noter Charles-François du Périer du Mouriez ${ }^{158}$, plus connu sous le nom de Dumouriez et vainqueur de la célèbre bataille de Valmy en 1792 mais aussi Charles du Périer, « prince des poètes lyriques » pour l'abbé Ménage, poète latin de la fin du XVII ${ }^{\mathrm{e}}$ siècle.

On peut également avancer une filiation avec François du Périer. Celle-ci n'est jamais explicite dans les documents que nous avons trouvés. Néanmoins, François du Périer lui succède dans la vie politique lyonnaise et surtout la division de l'héritage de Louis se fait entre Gaspard d'un côté et Marguerite Baronnat et François du Périer de l'autre, suggérant que François est le fils de Louis du Périer et de Marguerite Baronnat. Ses dates d'activité connues, les années 1530 et 1540, et son décès en 1557 laissent supposer qu'il s'agit d'un fils assez tardif qui a probablement au moins une dizaine voire une vingtaine d'années de moins que Gaspard. Contrairement à ce dernier, il reste à Lyon et est élu consul en 1537-1538, 15421543 et 1549-1550. Au début des années 1530, il est fermier du sel à Lyon et effectue quelques missions pour le consulat ${ }^{159}$. Il est alors désigné comme «noble homme, citoyen et bourgeois de Lyon ${ }^{160}$. Entre son second et son troisième mandat consulaire, il prend les titres d'écuyer et de seigneur d'Ouzielles. Ferdinand Frécon a retrouvé son testament où François institue comme héritiers universels Antoine et Claude Sanneton ${ }^{161}$, deux frères d'une autre famille consulaire lyonnaise avec laquelle il semble lié. Il laisse en outre une maison dans la rue Tramassac ${ }^{162}$ à Lyon à son neveu Pierre du Périer, troisième fils de Gaspard et fait apparemment des affaires à Paris : son testament est reçu au châtelet de Paris et il lègue une maison rue des fossés de Saint-Germain-l'Auxerrois à un procureur du parlement de Paris. On

\footnotetext{
${ }^{157}$ En 1528-1529, la maison sur la rue tirant du pont à Saint-Nizier est toujours à son nom (AML, CC39, fo 10$)$

${ }^{158}$ Dumouriez appartient à une branche cadette issue d'un frère de François du Périer, l'ami de Malherbe.

159 Jeanne-Marie Dureau, «En faveur de la chose publique » : délibérations consulaires de Lyon : 9 janvier 1533-22 décembre 1534 (n.s.), Lyon, Archives municipales, 1998, p. 502.

${ }^{160}$ AML, BB 52, $\mathrm{f}^{\circ} 150 \mathrm{r}^{\circ}$.

${ }^{161}$ Sur la famille Sanneton, et en particulier son activité de librairie, voir: Marie-Dominique Tamet, Les Senneton, marchands-libraires à Lyon au XVI $I^{e}$ siècle, mémoire d'étude pour le diplôme de conservateur de bibliothèque, ENSSIB, 2011. Antoine est conseiller du roi et avocat à Paris, délégué à la justice de Metz en 1558 et président de celle-ci en 1564. Claude Sanneton est un libraire lyonnais, seigneur du Magny-Fouchard et consul de Lyon en 1559-1560 et en 1563.

${ }^{162}$ Ce n'est donc pas la maison familiale où résidait Louis du Périer près de Saint-Nizier.
} 
peut donc supposer un mariage avec une femme de la famille Sanneton qui ne semble pas lui avoir laissé d'enfant. D'après les armoriaux consulaires lyonnais, François du Périer brise ses armes familiales en passant la bordure engrêlée de gueules à or.

Les généalogies de l'époque moderne donnent à Louis un autre fils, Jacques du Périer, chevalier de l'ordre de Malte, qui serait mort au siège de Rhodes en $1480^{163}$. César de Nostredame, familier de François du Périer petit-fils de Gaspard, atteste, au début du XVII siècle, avoir vu et lu des lettres de ce Jacques du Périer invitant son frère Gaspard à faire prendre la croix à l'un de ses fils ${ }^{164}$. Ce récit pose de nombreux problèmes. Il s'insère dans l'histoire des du Périer comme une preuve qu'ils sont d'ancienne noblesse puisque les chevaliers de l'ordre de Malte devaient normalement attester de plusieurs degrés de noblesse. Mais, sans fraude, un fils de Louis du Périer, bourgeois anobli mariée à une bourgeoise, ne peut apporter suffisamment de preuves de noblesse. De plus, en 1480, Gaspard du Périer a douze ans et Jacques, considéré comme son cadet peut difficilement en avoir plus de onze ans. Même en inversant l'ordre des frères, Jacques ne peut avoir plus de quatorze ans, Louis du Périer s'étant marié en 1465. L'ensemble de ces éléments rendent à peu près impossible qu'un fils de Louis du Périer ait pu être chevalier de Malte au siège de Rhodes de 1480. Les lettres vues par Nostredame sont probablement des faux à moins qu'il n'ait en fait rien vu mais brodé sur une anecdote rapportée par son protecteur. Comme la généalogie de la famille du Périer a été reconstruite en partie à l'époque moderne en intégrant des homonymes nobles, il est probable que ce soit le cas de ce Jacques du Périer, s'il a vraiment existé. D'autres généalogistes postérieurs donnent d'autres dates pour ce Jacques du Périer mais, sans preuve formelle, cela n'apporte que plus de confusion à l'affaire ${ }^{165}$.

Finalement, en plus d'avoir rendu à la société lyonnaise de la fin du $\mathrm{XV}^{\mathrm{e}}$ siècle un important bibliophile, cet article a permis de retracer la carrière d'un des plus importants Lyonnais de la seconde moitié $\mathrm{du} \mathrm{XV}$ siècle. Probablement issu de la famille Thomassin, une des plus importantes de la ville, Louis du Périer a servi pendant plusieurs décennies le roi, l'archevêque et la ville de Lyon. Ses talents furent reconnus par ses contemporains qui lui confièrent des charges, des honneurs et des mandats municipaux. Entré définitivement dans la noblesse à la toute fin $\mathrm{du} \mathrm{XV}^{\mathrm{e}}$ siècle, un quart de siècle après son ami Humbert de Varey, il peut également placer peu après son fils aîné au parlement d'Aix ce qui a contribué au déplacement de la famille du Périer en Provence au cours du XVI ${ }^{\mathrm{e}}$ siècle et son intégration dans la noblesse provençale. Si la charge de visiteur des gabelles de Provence était presque

\footnotetext{
${ }^{163}$ Les généalogies de l'époque moderne reprennent généralement le Martyrologe des chevaliers de S Iean de Hierusalem dits de Malte... de Mathieu de Goussancourt, imprimé en 1643. Jacques du Périer, fils de Louis, frère de Gaspard, inscrit avec les armes de notre famille du Périer, est dit mort au siège de Rhodes en 1680 [sic. Erreur probable pour 1480] (tome 2, p. 94).

${ }^{164}$ César de Nostredame, Histoire et chronique de Provence, Lyon, Simon Rigaud, 1624, p. 689.

165 Ferdinand Frécon semble dire qu'il a vu les preuves de noblesse produites par Jacques du Périer pour son entrée dans l'ordre, mais il n'est capable de ne citer que le nom d'un seul de ses grands-parents, Jean du Périer, barbier de Louis XI, dont il est à peu près certain qu'il n'a rien à voir avec la famille du Périer de Lyon. Pour lui, Jacques du Périer entre dans l'ordre en 1515 et meurt au siège de Rhodes en 1580 [sic]. Cette dernière erreur se trouvait déjà dans le Dictionnaire de la noblesse de François-Alexandre Aubert de La Chenaye-Desbois qui tente apparemment de corriger, de façon erronée, le Martyrologe de Goussancourt qu'il cite. Pour l'Armorial de Dauphiné de Gustave de Rivoire de la Bâtie, paru en 1867, Jacques devient Aymar et est chevalier de Malte en $1530 . .$. D'un point de vue purement chronologique, un tel Jacques du Périer ne pourrait mourir qu'au siège de Rhodes de 1522 mais aucun auteur ne mentionne cette éventualité.
} 
prémonitoire de cette translation vers le sud, elle a néanmoins trompé de nombreux auteurs qui ont cru à une origine provençale de Louis du Périer ou à une installation dans cette province dès son époque. Ses descendants ont joué là-dessus pour s'inventer une ascendance noble provençale remontant au XIV ${ }^{e}$ siècle grâce à des homonymes mais il n'en est rien. Cette confusion ainsi que son apparent isolement hors de toute famille consulaire lyonnaise avait contribué à rendre Louis du Périer peu visible dans les études sur la vie sociale et politique lyonnaise de la fin du Moyen Âge malgré une carrière riche de nombreuses missions et mandats et un rôle de premier plan au consulat. Cet article devrait réparer cette lacune et rendre à Louis du Périer toute son importance dans la cité rhodanienne.

\section{NOTICE BIO-BIBLIOGRAPHIQUE}

Jean-Benoît Krumenacker (CIHAM-UMR 5648) a soutenu une thèse en histoire médiévale en janvier 2019 intitulée Du manuscrit à l'imprimé : la révolution du livre à Lyon (1470-1520) sous la direction de Jean-Louis Gaulin (université Lyon 2) et de Dominique Varry (ENSSIB) avec le soutien d'un contrat doctoral. Il travaille sur les bibliothèques et les milieux intellectuels lyonnais de la fin du Moyen Âge ainsi que des débuts de l'imprimerie lyonnaise et a publié quelques articles et chapitres d'ouvrages sur ces sujets comme «Un Virgile plein de caractère », Gutenberg Jahrbuch, n ${ }^{\circ} 92,2017$; «Imprimer et traduire : Lyon au XV ${ }^{\mathrm{e}}$ siècle », Mémoires du livre, $\mathrm{n}^{\circ}$ 9/1, 2018 ou « Pierre Rostaing et Agobard au XVI siècle », in Marie-Céline Isaïa, François Bougard, Alexis Charansonnet (dir.), Lyon carolingien, Autour d'Agobard, Turnhout, Brepols, 2019 [à paraître].

\section{RESUME}

Louis du Périer, bibliophile bien connu des spécialistes des manuscrits enluminés du $\mathrm{XV}^{\mathrm{e}}$ siècle, est souvent présenté comme provençal du fait de son office de visiteur des gabelles à sel de Provence et de l'installation en Provence de sa descendance parmi laquelle on trouve le François du Périer à qui Malherbe adressa sa célèbre Consolation. Cet article vient montrer au contraire son ancrage lyonnais. Grâce aux archives lyonnaises, on peut ainsi retracer sa carrière dans la vie politique lyonnaise entre 1484 et 1503 . Il effectue plusieurs missions pour la ville et apparaît rapidement comme l'un des principaux Lyonnais dans la gestion des affaires communes pour lesquelles il est élu à quatre reprises au consulat. Il obtient de plus pour lui comme pour son fils aîné des offices royaux importants et acquiert la noblesse en 1498. La principale charge pour laquelle il est connu est celle de visiteur du sel de Provence, obtenue, semble-t-il, en 1486 mais réellement prise seulement en 1489. Néanmoins, les traces de ses activités lyonnaises rendent impossible un véritable exercice pratique de cette charge qui lui donne un certain rang dans les assemblées mais reste, d'une certaine façon, presque fictive. Son importante activité politique lyonnaise le rend proche de certains personnages comme Humbert de Varey et impose certainement son rattachement à une des meilleures familles lyonnaises de l'époque, probablement la famille Thomassin dont il serait un cadet.

Moyen Âge ; Lyon ; Provence ; Consulat ; Histoire urbaine

\section{ABSTRACT}

Louis du Périer is a well-known French bibliophile who commissioned several manuscripts illuminated by Lyon artists. He is also the great-grandfather of François du Périer to whom the poet Malherbe addressed one of the most famous French poems. But his life is almost unknown and his charge as chief of the salt administration (Visiteur des gabelles à sel) in Provence has often misled searchers. On the contrary, this article shows its roots 
in Lyon and its strong and lasting links with this city. Louis' origin and the first years of his life remain mysterious. He married a woman from Lyon in 1465 and served the king for a few years. In 1484, he began to serve the city of Lyon : he carries out missions to the king and to the Estates of Languedoc for the important question of the Lyon fairs. He was first elected Consul of Lyon in 1486 for two years and then served three more terms in 1490, 1497 and 1501. At that time, he was one of the most important people in Lyon. He is one of the first in the order of consuls and is regularly consulted for the administration of the city when he is not elected. He obtained for himself and for his son many municipal honours. In addition, he became chief of the salt administration in Provence in 1489, was appointed courrier (chief for civil justice in Lyon) to the archbishop around 1493 and obtained nobility in 1498. Thanks to him, his son Gaspard du Périer became a member of the Aix parliament when it was created in 1501. This office will then lead to the relocation of the du Périer family to Provence in the $16^{\text {th }}$ century. Louis du Périer died around 1506. Several of his social relationships are known. He was close to the Varey family, the most important consular family in Lyon in the $15^{\text {th }}$ century. He is thus the guardian of Antoine de Varey, the heir of this family. But other elements, in particular his coat of arms, suggest a relationship with another important family in Lyon, that of the Thomassin. He is probably a cadet of this family whose members served the dauphin, the king and the city of Lyon in the $15^{\text {th }}$ century. He probably has successively two or three wives and at least two sons Gaspard and François. All this information makes it possible to draw a portrait of a member of Lyon's bourgeois elite at the end of the Middle Ages.

Middle Ages ; Lyon ; Provence ; Consulate ; Urban history 